\title{
A review of Cyclidiinae from China (Lepidoptera, Drepanidae)
}

\author{
Nan Jiang', Shuxian Liu',2, Dayong Xue', Hongxiang Han'
}

I Key Laboratory of Zoological Systematics and Evolution, Institute of Zoology, Chinese Academy of Sciences, Beijing 100101, China 2 University of the Chinese Academy of Sciences, Beijing 100049, Chin

Corresponding author: Hongxiang Han (hanhx@ioz.ac.cn)

Academic editor: E. van Nieukerken | Received 1 August 2015 | Accepted 2 December 2015 | Published 14 January 2016

http://zoobank.org/442C6C2F-356C-42E5-B63F-F2931DE34683

Citation: Jiang N, Liu S, Xue D, Han H (2016) A review of Cyclidiinae from China (Lepidoptera, Drepanidae). ZooKeys 553: 119-148. doi: 10.3897/zookeys.553.6153

\begin{abstract}
The subfamily Cyclidiinae from China is reviewed: two genera and seven species are reported from China. One new subspecies, Cyclidia fractifasciata indistincta subsp. n., is described. Two new synonyms are established: Cyclidia substigmaria (Hübner, 1831) (= Cyclidia substigmaria brunna Chu \& Wang, 1987, syn. n. = Cyclidia tetraspota Chu \& Wang, 1987, syn. n.). One misidentification in Chu \& Wang (1987) is corrected. Identification keys and diagnoses for all discussed Chinese species are provided. External features and genitalia are depicted. In addition, results of DNA barcoding for five taxa of Cyclidia are briefly discussed.
\end{abstract}

\section{Keywords}

DNA barcodes, morphology, new subspecies, new synonymy, taxonomy 


\section{Introduction}

The subfamily Cyclidiinae Warren, 1922, is the smallest subfamily within four subfamilies (besides Drepaninae, Oretinae, and Thyatirinae) of Drepanidae. This subfamily was first proposed as Eucherinae by Strand (1911) based on the genus Euchera Hübner, 1825. Later, it was treated as a separate family (Inoue 1962), followed by other authors (Fletcher 1979, Chu and Wang $(1987,1991)$ (Yan et al. 2009). However, Minet (1983) regarded Cyclidiinae as a subfamily of Drepanidae, based on the study of the tympanal organs. This treatment was later followed by many researchers, e.g. Holloway (1998) and Minet (2002), and was also supported by molecular data (Wu et al. 2010).

Recently, Chen (2011) performed a phylogenetic analysis of Cyclidiinae, based on morphological characters. In his study, the monophyly of respectively Cyclidiinae, Cyclidia and Mimozethes was supported, and most synapomorphies for Cyclidiinae proposed by previous studies were shown to be plesiomorphies. Three major synapomorphies of Cyclidiinae were given (Chen 2011): 1) the developed anterotergal syndeses (a paired of semi-translucent structure, see Yan et al. 2005) at the anterior margin of the $2^{\text {nd }}$ tergum; 2) a pair of androconial hair-pencils on the $2^{\text {nd }}$ pleuron of the male and 3) the short and robust gnathos in the male genitalia.

Species of Cyclidiinae are distributed in the Palearctic Asia and Oriental regions. Up to the present, two genera (Cyclidia Guenée, 1858 and Mimozethes Warren, 1901) have been recognized in Cyclidiinae. Ten species and eight subspecies are included in Cyclidia, with six species and four subspecies (C. substigmaria substigmaria (Hübner, 1831), C. substigmaria brunna Chu \& Wang, 1987, C. substigmaria intermedia Prout, 1918, C. tetraspota Chu \& Wang, 1987, C. rectificata rectificata (Walker, 1862a), C. fractifasciata (Leech, 1898), C. sericea Warren, 1922, C. orciferaria Walker, 1860) recorded in China (Moore 1886, Aurivillius 1894, Swinhoe 1899, Strand 1911, Warren 1914, Bryk 1943, Inoue 1962, Chu and Wang 1991, Chang 1989, Holloway 1998, Lutz and Kobes 2002). Three species are included in Mimozethes, with two species recorded in China, M. lilacinaria (Leech, 1897) and M. angula Chu \& Wang, 1987. However, the taxonomy of some Chinese taxa remained unclear ( e.g. the subspecies delimitation of C. substigmaria; the taxonomic status of C. tetraspota and the puzzling distribution of $C$. sericea) (Yan et al. 2009, Chen 2011). It is obviously that further research is needed and molecular markers could be used to clarify these problems.

The DNA barcoding method using a 658 bp base pair fragment of the cytochrome c oxidase subunit I gene (COI) as a tool for species discrimination was first put forward based on two hundred closely related species of Lepidoptera (Hebert et al. 2003). It has since been successfully used for species delimitation in lepidopteran species that are difficult to separate morphologically (see Hajibabaei et al. 2006, Yang et al. 2012). The barcoding gap between intra- and inter-specific variation was used for species 
discrimination (Hebert et al. 2004a, Meier et al. 2006, Meier et al. 2008, Sihvonen et al. 2014, Jiang et al. 2014).

In the present study an overview of the Chinese Cyclidiinae is given with diagnostic characters for each genus and species, one new subspecies is described, two new synonyms are established, and one misidentification in Chu and Wang (1987) is revised. Also photos of external features and genitalia are provided of all Chinese species discussed. In addition, we discuss the application of the results of DNA barcoding for delimitation of five taxa of Cyclidia. As a result of this study five species and five subspecies of Cyclidia and two species of Mimozethes are regarded as valid for the fauna of China.

\section{Materials and methods}

Morphology. Studied specimens mainly belong to the Institute of Zoology, Chinese Academy of Sciences, Beijing, China (IZCAS) and the Natural History Museum, London, United Kingdom BMNH. Terminology for wing venation follows the ComstockNeedham System (Comstock 1918), and that of the genitalia is based on Klots (1970), Nichols (1989) and Kristensen (2003). Photographs of the moths were taken with digital cameras. Composite sharp images were generated using Auto-Montage software version 5.03.0061 (Synoptics Ltd). The plates were compiled using Adobe Photoshop software.

DNA-Barcoding. Prior to DNA sequencing, one or two legs were removed from several specimens of each of five examined taxa (C. substigmaria substigmaria, C. rectifcata rectificata, C. fractifasciata fractifasciata, C. fractifasciata indistincta, C. orciferaria). DNA extraction was done using Qiagen DNeasy Blood and Tissue Kit (Qiagen, Beijing, China). The primers for the amplification of the $658 \mathrm{bp}$ fragment were LepF1 (5'-ATTCAACCAATCATAAAGATATTGG-3'), LepR1 (5'-TAAACTTCTGGATGTCCAAAAAATCA-3') (Hebert et al. 2004a). The PCR reactions were performed using the standard procedure described by Hebert et al. (2004a). The PCR products were detected by $1 \%$ agarose gel electrophoresis and directly sequenced with ABI PRISM 3730xl capillary sequencers. The amplification and sequencing for some dried material (Sequence ID begins with "DB") were carried out in BGI-Shenzhen, (China) using standard protocols described in Hebert et al. (2004a). Forward and reverse nucleotide sequences were assembled in SeqMan 5.01 (DNASTAR, Inc. 1996). The assembled sequences were aligned and manually edited in MEGA 5.0 (Tamura et al. 2011). The neighbor-joining (NJ) tree (Saitou and Nei 1987) was reconstructed based on Kimura 2-parameter (K2P) distances (Kimura 1980) using MEGA 5.0. All the sequences have been deposited in GenBank under accession numbers, and their full data including images and are in the Barcode of Life Database (http://www.boldsystems.org; see Ratnasingham and Hebert 2007) (Table 1). 
Table I. Cyclidia species included in this study with GenBank accession numbers and BOLD process ID.

\begin{tabular}{|c|c|c|c|c|c|}
\hline Taxa & Sequence ID & Collecting locality & $\begin{array}{c}\text { Collecting } \\
\text { date }\end{array}$ & $\begin{array}{c}\text { GenBank } \\
\text { accession no. }\end{array}$ & $\begin{array}{c}\text { BOLD } \\
\text { process ID }\end{array}$ \\
\hline \multirow{18}{*}{$\begin{array}{l}\text { C. substigmaria } \\
\text { substigmaria }\end{array}$} & $\mathrm{D}$ & $\begin{array}{l}\text { West Tianmushan, } \\
\text { Zhejiang }\end{array}$ & Jul. 2003 & KR872896 & CLDC001-15 \\
\hline & $\begin{array}{l}\text { DB00173 } \\
\text { DB00174 } \\
\end{array}$ & $\begin{array}{c}\text { Wuzhishan, Hainan } \\
\text { Lingshui, Hainan }\end{array}$ & $\begin{array}{l}\text { May } 2007 \\
\text { May } 2007 \\
\end{array}$ & $\begin{array}{l}\text { KR872897 } \\
\text { KR872898 }\end{array}$ & $\begin{array}{l}\text { CLDC002-15 } \\
\text { CLDC003-15 } \\
\end{array}$ \\
\hline & DB00181 & Baotianman, Henan & Aug. 2008 & KR872899 & CLDC004-15 \\
\hline & DB00182 & Luoyang, Henan & Aug. 2006 & KR872900 & CLDC005-15 \\
\hline & DB00184 & Baoshan, Yunnan & Aug. 2007 & KR872901 & CLDC006-15 \\
\hline & DB00189 & Yanling, Hunan & Jul. 2008 & KR872902 & CLDC007-15 \\
\hline & IOZ LEP M 01129 & Mengla, Yunnan & Jul. 2013 & KR872903 & CLDC008-15 \\
\hline & IOZ LEP M 01134 & Tengchong, Yunnan & Aug. 2013 & KR872904 & CLDC009-15 \\
\hline & IOZ LEP M 01304 & $\begin{array}{l}\text { West Tianmushan, } \\
\text { Zhejiang }\end{array}$ & Jul. 2011 & KR872905 & CLDC010-15 \\
\hline & IOZ LEP M 08961 & Mengla, Yunnan & Jul. 2013 & KR872906 & CLDC011-15 \\
\hline & IOZ LEP M 09195 & Qushi, Yunnan & Aug. 2013 & KR872907 & CLDC012-15 \\
\hline & IOZ LEP M 16605 & Kangxian, Gansu & Aug. 2014 & KR872908 & CLDC013-15 \\
\hline & IOZ LEP M 16606 & Kangxian, Gansu & Aug. 2014 & KR872909 & CLDC014-15 \\
\hline & IOZ LEP M 16607 & Kangxian, Gansu & Aug. 2014 & KR872910 & CLDC015-15 \\
\hline & IOZ LEP M 16608 & Kangxian, Gansu & Aug. 2014 & KR872911 & CLDC016-15 \\
\hline & IOZ LEP M 17993 & Liuku, Yunnan & Sep. 2014 & KR872912 & CLDC017-15 \\
\hline & IOZ LEP M 17994 & Liuku, Yunnan & Sep. 2014 & KR872913 & CLDC018-15 \\
\hline & IOZ LEP M 02790 & Guilin, Guangxi & Apr. 1952 & KR872914 & CLDC019-15 \\
\hline \multirow{7}{*}{$\begin{array}{l}\text { C. rectificata } \\
\text { rectificata }\end{array}$} & DB00226 & Bomi, Tibet & Aug. 2005 & KR872923 & CLDC020-15 \\
\hline & DB00228 & Mêdog, Tibet & Aug. 2006 & KR872924 & CLDC021-15 \\
\hline & DB00229 & Mainling, Tibet & Aug. 2006 & KR872925 & CLDC022-15 \\
\hline & IOZ LEP M 03475 & Zayü, Tibet & Aug. 2014 & KR872926 & CLDC023-15 \\
\hline & IOZ LEP M 03476 & Zayü, Tibet & Aug. 2014 & KR872927 & CLDC024-15 \\
\hline & IOZ LEP M 03477 & Zayü, Tibet & Aug. 2014 & KR872928 & CLDC025-15 \\
\hline & IOZ LEP M 16015 & Zayü, Tibet & Aug. 2014 & KR872929 & CLDC026-15 \\
\hline \multirow{4}{*}{$\begin{array}{l}\text { C. fractifasciata } \\
\text { fractifasciata }\end{array}$} & IOZ LEP M 00657 & Pianma, Yunnan & May 2011 & KR872930 & CLDC027-15 \\
\hline & IOZ LEP M 00683 & Pianma, Yunnan & May 2011 & KR872931 & CLDC028-15 \\
\hline & IOZ LEP M 07012 & Pianma, Yunnan & May 2011 & KR872932 & CLDC029-15 \\
\hline & IOZ LEP M 07013 & Pianma, Yunnan & May 2011 & KR872933 & CLDC030-15 \\
\hline \multirow{5}{*}{$\begin{array}{l}\text { C. fractifasciata } \\
\text { indistincta }\end{array}$} & IOZ LEP M 16601 & Kangxian, Gansu & Aug. 2014 & KR872934 & CLDC031-15 \\
\hline & IOZ LEP M 16602 & Kangxian, Gansu & Aug. 2014 & KR872935 & CLDC032-15 \\
\hline & IOZ LEP M 16603 & Kangxian, Gansu & Aug. 2014 & KR872936 & CLDC033-15 \\
\hline & IOZ LEP M 16604 & Kangxian, Gansu & Aug. 2014 & KR872937 & CLDC034-15 \\
\hline & IOZ LEP M 09387 & Wushan, Chongqing & Jul. 2013 & KT250118 & CLDC035-15 \\
\hline \multirow{8}{*}{ C. orciferaria } & DB00202 & Bawangling, Hainan & May 2007 & KR872915 & CLDC036-15 \\
\hline & DB00203 & Wuzhishan, Hainan & Apr. 2008 & KR872916 & CLDC037-15 \\
\hline & DB00210 & Yanling, Hunan & Jul. 2008 & KR872917 & CLDC038-15 \\
\hline & DB00211 & Yanling, Hunan & Jul. 2008 & KR872918 & CLDC039-15 \\
\hline & DB00213 & Shixing, Guangdong & Jun. 2008 & KR872919 & CLDC040-15 \\
\hline & DB00216 & Baoshan, Yunnan & Aug. 2007 & KR872920 & CLDC041-15 \\
\hline & IOZ LEP M 01208 & $\begin{array}{l}\text { West Tianmushan, } \\
\text { Zhejiang }\end{array}$ & Jul. 2011 & KR872921 & CLDC042-15 \\
\hline & IOZ LEP M 01324 & $\begin{array}{c}\text { West Tianmushan, } \\
\text { Zhejiang }\end{array}$ & Jul. 2011 & KR872922 & CLDC043-15 \\
\hline
\end{tabular}




\section{Results}

\section{Taxonomy}

\section{Cyclidiinae Warren, 1922}

Cyclidiinae Warren, 1922: 444.

\section{Cyclidia Guenée, 1858}

Cyclidia Guenée, 1858: 62. Type species: Cyclidia substigmaria (Hübner, 1831), by monotypy.

Nelcynda Walker, 1862a: 1142. Type species: Nelcynda rectificata Walker, 1862, by monotypy.

Ciclidia Chou \& Xiang, 1984: 159. [Incorrect spelling of Cyclidia Guenée.]

Generic characters. Head. Antennae lamellate, partly unipectinate, rami very short (Fig. 1a). Frons not protruding. Labial palpi with third segment distinct, up-curved. Thorax. Hind tibia with two pairs of spurs. Apex of forewing often rounded, sometimes pointed and protruding. Wing colour usually white or grey (except $C$. orciferaria); antemedial and postmedial lines of forewing double; medial line of forewing broad; terminal lines of both wings usually double, sometimes single (e.g. $C$. substigmaria, C. rectificata, and C. diehli Lutz \& Kobes, 2002). Venation (Fig. 3a). Forewing with $R_{1}$ separate, $R_{2-4}$ and $R_{5}$ stalked, $R_{2}$ and $R_{3+4}$ stalked, $R_{5}$ and $M_{1}$ separate, $M_{2}$ arising from middle of discocellulars; Hind wing with $S_{c}+R_{1}$ close to Rs beyond distal cell, then far from Rs, $\mathrm{M}_{2}$ arising from middle of discocellulars. Anterotergal syndeses developed at anterior margin of $2^{\text {nd }}$ tergum (Fig. 2). A pair of androconial hair-pencils present on $2^{\text {nd }}$ sternum of male (Fig. 2). Male genitalia. Uncus triangular; socii developed, often sclerotized (except $C$. orciferaria), sometimes with small setose process at base (e.g. C. pitimani (Moore, 1886), C. sericea and C. diehli); gnathos connected at middle and with median process narrow and triangular; valva simple and broad; juxta deeply concaved posteriorly; saccus short and broad, rounded terminally; phallus slightly curved; vesica without cornuti. Female genitalia. Papillae anales broad and rounded; lamella postvaginalis usually well developed; ductus bursae very long and narrow, with a colliculum; corpus bursae oval, with a paired band-like spinose signa.

Diagnosis. Cyclidia is quite different from Mimozethes externally and in the genitalia. For example, externally, the rami of the antennae are much shorter; the species of Cyclidia are much larger, and the postmedial lines of forewing are often double, while in Mimozethes, it is single and forms a ">" shaped protrusion near $\mathrm{R}_{5}$; in the male genitalia, the socii are well developed in Cyclidia, but absent in Mimozethes; the sacculus unmodified in Cyclidia but forming a process in Mimozethes; in the female genitalia, the signa are a paired band-like sclerotization in Cyclidia, but absent in Mimozethes.

Distribution. China, Japan, Korean Peninsula, south and southeast Asia. 


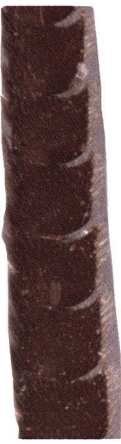

1a

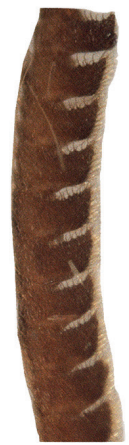

$1 \mathrm{~b}$

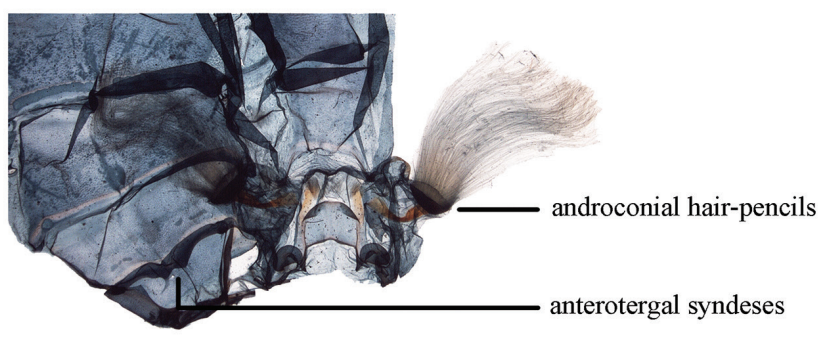

2

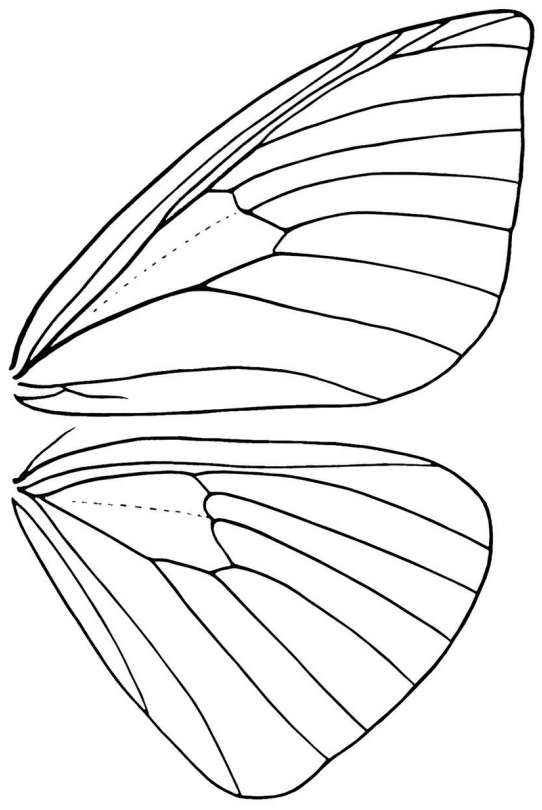

$3 a$

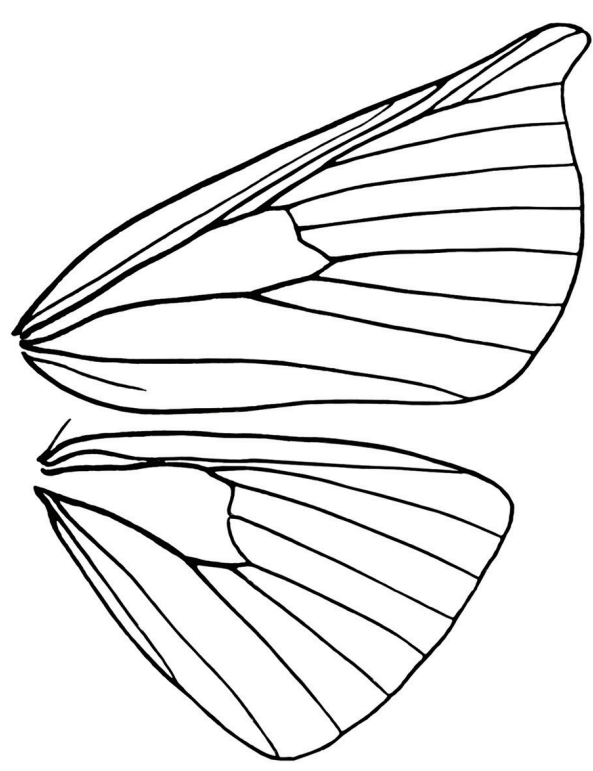

$3 b$

Figures I-3. I Male antennae a Cyclidia substigmaria b Mimozethes angula $\mathbf{2}$ Anterotergal syndeses and androconial hair-pencils 3 Wing venation (from Chu and Wang, 1991) a C. substigmaria b M. angula.

\section{Key to Chinese Cyclidia species}

1 Wings colour white or grey 2

- Wings colour blackish brown C. orciferaria, Figs 18-19

2 Discal spots on hind wing distinct 3

- Discal spots on hind wing indistinct

3 Discal spots on hind wing dark grey.. C. substigmaria substigmaria, Figs 4-8

- $\quad$ Discal spots on hind wing black C. substigmaria intermedia, Fig. 9 


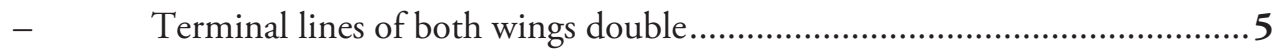

5 Outer margin of forewing medial line forming an right angle below $\mathrm{M}_{3} \ldots .66$

- $\quad$ Outer margin of forewing medial line not forming an right angle below $\mathrm{M}_{3}$.

C. pitimani, Figs 12-13

6 Outer line of antemedial line and inner line of postmedial line of forewing distinct .................................. C. fractifasciata fractifasciata, Figs 14-15

- $\quad$ Outer line of antemedial line and inner line of postmedial line of forewing invisible

C. fractifasciata indistincta, Figs 16-17

\section{Cyclidia substigmaria (Hübner, 1831)}

Euchera substigmaria Hübner 1831: 29. pl. 90, figs 519, 520. Syntypes, China. Cyclidia substigmaria: Guenée 1858: 63.

Abraxas capitata Walker, 1862a: 1121. Holotype +, China: Hong Kong (BMNH).

Euchera capitata: Strand 1911: 196.

Cyclidia substigmaria brunna Chu \& Wang, 1987: 205. Holotype ${ }^{7}$, China: Sichuan:

Emeishan, Qingyinge (IZCAS). Syn. n.

Cyclidia tetraspota Chu \& Wang, 1987: 206. Holotype Ô, China: Yunnan: Xishuangbanna, Yunjinghong (IZCAS). Syn. n.

Diagnosis. In external appearance, this species is distinguishable from other congeners by the following characters: the discal spots of hind wing are very distinct on the upper side and the underside; the discal spot of the forewing is covered with white scales on the upper side; two greyish brown markings are present inside the anal angle of the forewing. The male genitalia of the species are close to those of $C$. rectificata, but the terminal part of the uncus and the socii are narrower; the vesica is much more scobinate. In the female genitalia, the two signa are close to each other posteriorly, while in C. rectificata, they are almost parallel.

Remarks. There are five subspecies of $C$. substigmaria:

C. s. substigmaria (Hübner, 1831), most parts of China and Vietnam;

C. s. intermedia Prout, 1918 in Tibet;

C. s. nigralbara Warren, 1914 in Japan and Korean Peninsula;

C. s. modesta Bryk, 1943 in Myanmar;

C. s. superstigmaria Prout, 1918 in India and Nepal.

Distribution. China, Japan, Korean Peninsula, India, Nepal, Myanmar, Vietnam.

Biological notes. Sugi (1987) and Holloway (1998) mentioned that larval Cyclidiinae may be uniquely associated with the plant family Alangiaceae (now incorporated in Cornaceae). However, Cyclidia substigmaria also has been recorded from Malvaceae (Hibiscus cannabinus L.) (Chu 1981, Chu and Wang 1987, 1991, Kadoorie Farm and Botanical Garden 2004). The morphology of the eggs, larva, pupa and life history of C. substigmaria were described in detail by Zhou and Wang (1985), Chu and Wang (1991) and Yan et al. (2009). 


\section{Cyclidia substigmaria substigmaria (Hübner, 1831)}

Figs 4-8, 24-28, 37-39, 48-50

Diagnosis. The subspecies is very similar to C. substigmaria intermedia, but differs externally by the paler discal spot of the hind wing and the two less distinct markings inside the anal angle of the forewing.

Type material examined. CHINA: Sichuan (IZCAS): $1 \delta^{\Uparrow}$ (Holotype of $C$. substigmaria brunna), Emeishan, Qingyinge, 800-1000 m, 17.V.1957, coll. Wang Zongyuan. Zhejiang (IZCAS): 1 ( (Allotype of C. substigmaria brunna), Hangzhou, 4.V.1975, coll. Zhang Baolin. Fujian (IZCAS): $3{ }^{\lambda}$ (Paratypes of C. substigmaria brunna), Wuyishan, 6-21.V.1983, coll. Wang Linyao. Yunnan (IZCAS): 1 + (Paratypes of C. substigmaria brunna), Liuku, 2500 m, 23.V.1981, coll. Liao Subai;

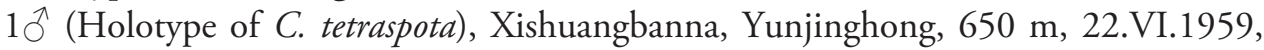
coll. Meng Xuwu; 1 (ㅅ (Allotype of C. tetraspota), Yiwubanna, Menglun, $650 \mathrm{~m}$, 23.VII.1959, coll. Zhang Facai; $1 \hat{\sigma}^{\lambda}$ (Paratype of C. tetraspota), ibidem, 28.V.1958, coll. Wang Shuyong. Hainan (IZCAS): $1 \delta^{\lambda}$ (Paratype of C. tetraspota), Wanning, 10 m, 9.IV.1960, coll. Li Zhenfu. Guangxi (IZCAS): $1 \delta^{\lambda}$ (Paratype of C. tetraspota), Guilin, Liangfeng, 20.IV.1952. Hongkong (BMNH): 1 + , collector and collecting date unknown (Holotype of $C$. substigmaria capitata).

Additional material examined. CHINA: Henan (IZCAS): 10, Luoyang, Huaguoshan, 4.VIII.2006, coll. Song Hao; 1 ㅇ, Baiyunshan, 1400 m, 27.VII.2003,

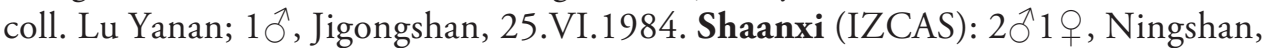
Guanghuojie, 1189 m, 28.VII.2014, coll. Liu Shuxian and Ban Xiaoshuang; 10, Zhashui, Yingpanzhen, 980 m, 31.VII.2014, Liu Shuxian and Ban Xiaoshuang; $1 \widehat{\jmath}$, Xunyang, Bailiuzhen, 386 m, 3.VIII.2014, coll. Liu Shuxian and Ban Xiaoshuang. Gansu (IZCAS): 10, Wenxian, Qiujiaba, 2200-2350 m, 29.VI.1998, coll. Yuan Decheng; 1우 Kangxian, Baiyunshan, 1250-1750 m, 12.VII.1998, coll. Wang Shuyong; $1 \jmath^{\top} 7$, Kangxian, Yangba, Meiyuangou, 1000 m, 13.VIII.2014, coll. Xue Dayong \& Ban Xiaoshuang; 1q, Wenxian, Lukou, 22.V.1987. Jiangsu (IZCAS): $7 \AA 4 q$, Chemo, 22.IV-2.V.1935, coll. O. Piel. Anhui (IZCAS): 19 , Linzongchang, IX.1970, coll. Mai Weiqiang; 2 9 , Yuexi, Linyeju, 11.IX.1982, coll. Zhou Tiying. Zhejiang (IZCAS): $5{ }^{\lambda} 3$ + , Lin'an, West Tianmushan, 400-1506 m, 6.IX.1981, 26-30.VII.2003, 27.VII.2011, coll. Xue Dayong et al.; 15ð1우, Tianmushan, 15-25.VI.1936, 25-30.VIII.1947, 22.VIII.1972, 28-31.VII.1998, coll. O. Piel et al.; $1 \delta^{\Uparrow} 1$, Hangzhou, 4.V.1975, 1981, coll. Zhang Baolin; 1 ${ }^{\lambda}$, Qingyuan, Fengyangshan, Datianping, 1290 m, 6-10.VIII.2003, coll. Han Hongxiang. Hubei (IZCAS): 1ठึ, Shennongjia, Muyu, 22.VII.1998, coll. Zhou Hongzhang; 1옹 Shennongjia, Dalongtan, $2700 \mathrm{~m}, 27$. VII.1998, coll. Zhou Haisheng; 10, Xingshan, Longmenhe, 1300 m, 12.IX.1994, coll. Song Shimei; 4ㅇ, Xuan'en, 650 m, 25.V.1989, coll. Li Wei; 1 , Hefeng, Fenshuiling Linchang, 31.VII.1989, coll. Li Wei. Jiangxi (IZCAS): 19, Yifeng, Yuanqian, 8.IX.1959. Hunan (IZCAS): 1 , Yanling, Taoyuandong, 631 m, 4-8.VII.2008, coll. Chen Fuqiang; 1, Fenghuang, 15.IX.1988, coll. Song Shimei; 1, Cili, 3.IX.1988, coll. Song Shimei. 

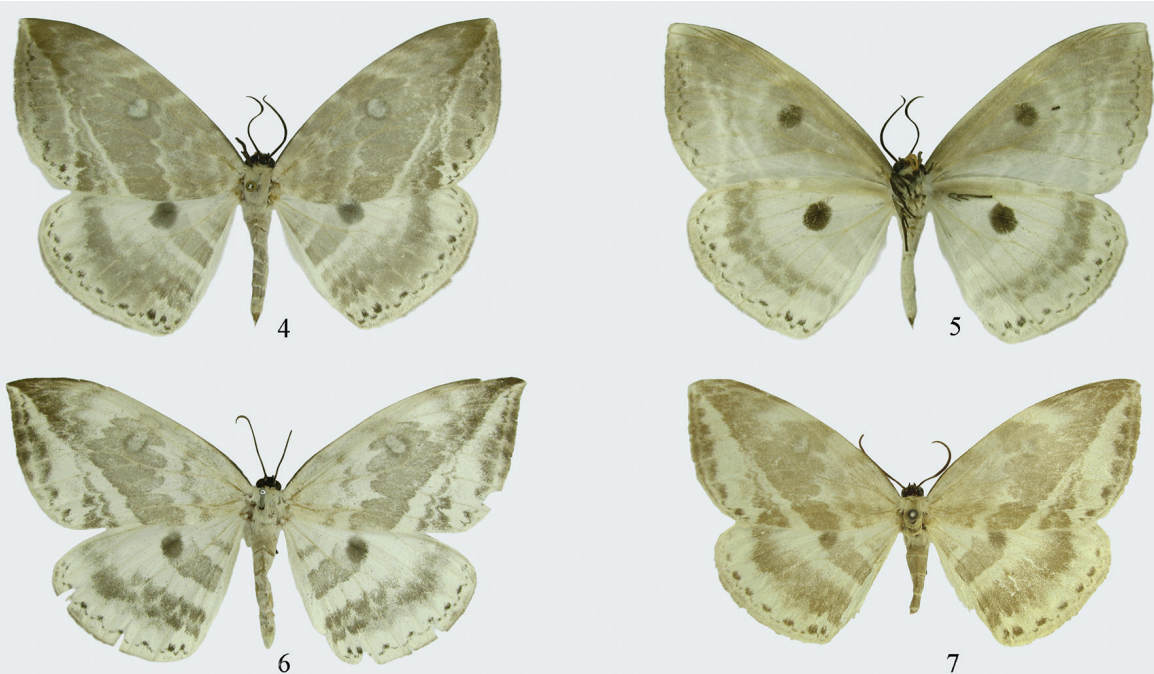

7
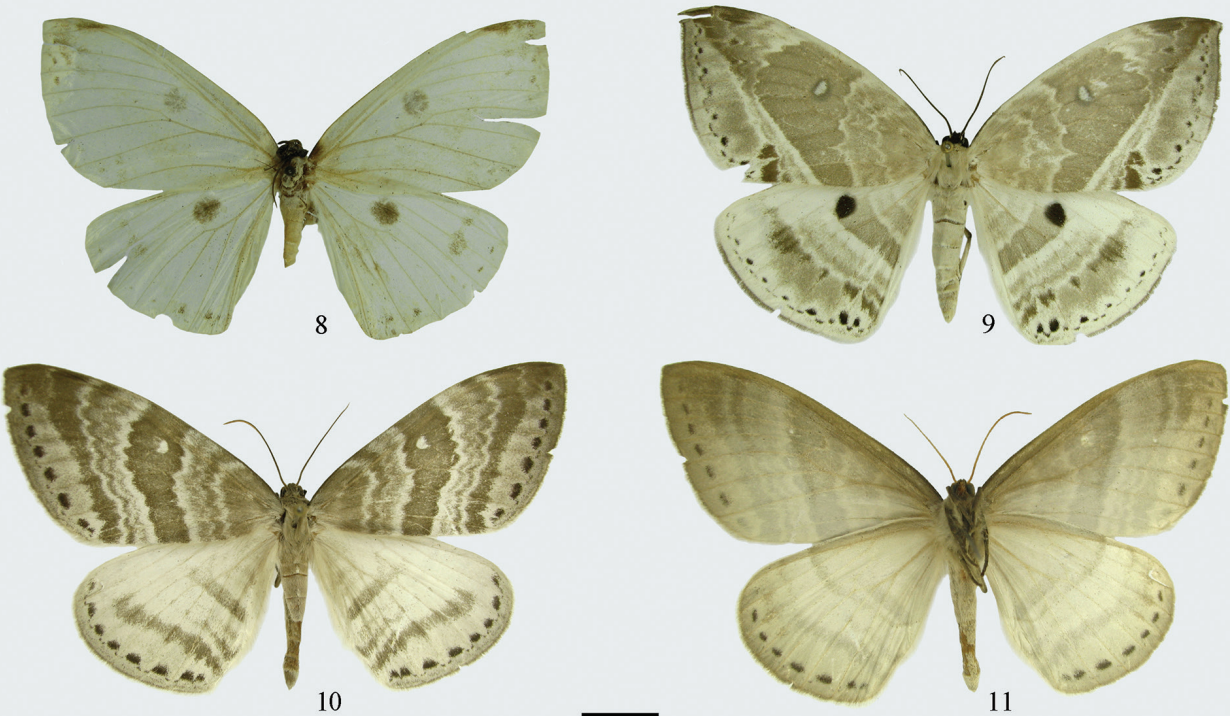

Figures 4-I I. Adults. 4-9 Cyclidia substigmaria substigmaria 4 male (with dot-like and wavy submarginal line of the forewing, Yunnan) $\mathbf{5}$ ditto, underside $\mathbf{6}$ male (with faint, broad and interrupted submarginal line of the forewing, Zhejiang) $\mathbf{7}$ male (holotype of C. substigmaria brunna, Sichuan) 8 male (holotype of C. tetraspota, Yunnan) 9 C. substigmaria intermedia, male (Tibet) I0-II C. rectificata $\mathbf{1 0}$ male (Tibet) I I ditto, underside. Scale bar: $1 \mathrm{~cm}$.

Fujian (IZCAS): 11 $\delta^{\Uparrow} 99$, Wuyishan, 26.IV-14.VI.1983, coll. Wang Linyao and Zhang Baolin; $1 \overbrace{}^{\Uparrow}$, Xinkou, 15.VI.1981, coll. Lin Yibiao; $2{ }^{\lambda} 1$ 우, Jianyang, Huangkeng, 270-950 m, 23.IV-1.V.1960, coll. Jiang Shengqiao and Zuo Yong; 1우, Chong'an, Xingcun, Guadun, 840-1210 m, 25.VIII.1960, coll. Ma Chenglin; 1ㅇ, Chong'an, Xingcun, Sangang, 740 m, 17.V.1960, coll. Zhang Yiran. 
Guangdong (IZCAS): 1 $\hat{\jmath}$, Guangzhou, 8.VI.1973, coll. Zhang Baolin; 4 $\jmath^{\lambda}+$, Guangzhou, Sanyuanli, 27.IV.1958, coll. Wang Linyao. Hainan (IZCAS): Wan-

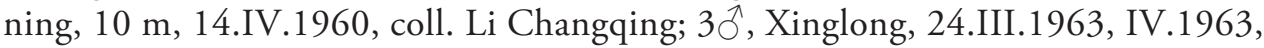

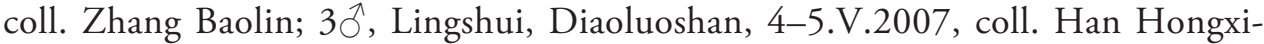
ang; 1ㅇ, Wuzhishan, Shuiman, 600 m, 12.V.2007, coll. Han Hongxiang; 1우

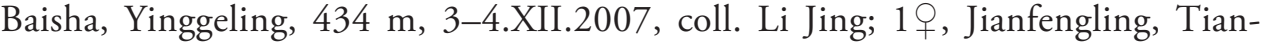
chi, 3.III.1982, coll. Long Yongcheng. Guangxi (IZCAS): $10^{\lambda} 1$ 엉 Jinxiu, Luoxiang, 200-400 m, 1-16.V.1999, coll. Huang Fusheng and Han Hongxiang; 19, Jinxiu, Yonghe, 500 m, 12.IV.1999, coll. Han Hongxiang; 19, Jinxiu, Jinzhong Gonglu, 1100 m, 12.V.1999, coll. Li Wenzhu; 20, Guilin, Yanshan, 26.IX.1958, 19.XI.1959; 10^5우, Fangcheng, Fulong, 240-260 m, 1.III.1998, 19-20.IV.1998, coll. Li Wenzhu and Wu Chunsheng; 1ð̃, Napo, Nianjing, 900 m, 11.IV.1998, coll. Wu Chunsheng; 1, Napo, Defu, 1350 m, 19.VI.2000, coll. Yao Jian; 1 , Napo, Nonghua, 990 m, 13.IV.1998, coll. Li Wenzhu; 19, Napo, Baihe, 540 m, 8.IV.1998, coll. Qiao Gexia; 10, Pingxiang, 230 m, 8.VI.1976, coll. Zhang Baolin; 2q, Longsheng, 10-11.VI.1980, coll. Zhong Tiesen and Song Shimei; 2ㅇ, Daxin, Xialei, 680 m, 31.III.1998, coll. Li Wenzhu; 20, Longzhou, Nonggang, 195 m, 15-17.VII.2013, coll. Liu Shuxian and Li Xinxin. Sichuan (IZCAS): 1ㅇ, Emeishan, Baoguosi, 550-750 m, 8.IV.1957, coll. Wang Zongyuan;

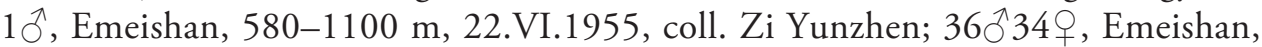
Qingyinge, 800-1000 m, 17.IV-20.V.1957, 19.IX-28.X.1957, coll. Zhu Fuxing et al.; 19, Yanyuan, Jinhe, 2.VII.1984, coll. Chen Yixin. Guizhou (IZCAS): 19, Sinan, 350 m, 9.V.1983, coll. Liu Yanxian; 10̄, Koei-Yang, 5.IX.1935. Yunnan (IZCAS): 2ð77 , Xishuangbanna, Mengna, $550 \mathrm{~m}, 22-30 . V I .1959$, coll. Zhang

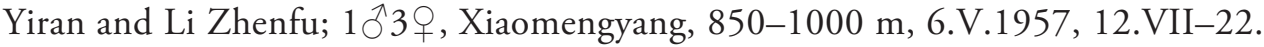
VIII.1957, 10.X.1957, coll. Wang Shuyong et al.; 1ð1우, Xishuangbanna, Menghun, 160-750 m, 4.VI.1958, coll. Meng Xuwu et al.; 1우, Xishuangbanna, Yunjinghong, $650 \mathrm{~m}$, 3.VII.1957, coll. Wang Shuyong; $2 \delta 6$, Xishuangbanna, Mengla, 620-650 m, 2.V-6.VI.1959, coll. Zhang Yiran et al.; 6\$9우, Mengla, Menglun, 650-665 m, 22-29.X.1958, 3.IV-18.V.1964, 29.VII.2013, coll. Wang Shuyong et al.; 10, Xishuangbanna, Menghai, 1200-1600 m, 18.VII.1958, coll. Wang Shuyong; 2ㅇ, Xishuangbanna, Ganlanba, 560 m, 9-10.VII.1958, coll. Li Chuanlong; 10, Xishuangbanna, Bubang, 700 m, 14.IX.1993, coll. Yang Longlong; 1우, Xishuangbanna, Yiwu, 800-1300 m, 13.VII.1959, coll. Pu Fuji; 6 ${ }^{\lambda} 1$, , Baoshan, Baihualing, $1520 \mathrm{~m}$, V.11-13.VIII.2007, coll. Wu Chunguang and Lang Songyun; 201 \% , Baoshan, Bawan, 1040-1100 m, 19-23.1992, 8-10.VIII.2007, 8-10. VIII.2013, coll. Wu Chunguang et al.; 2ð3 2 , Baoshan, Xinujiang Hegu, 8001000 m, 10-11.V.1955, coll. Xue Yufeng; 10, Tengchong, Qushi, Dabacun, 1873 m, 4.VIII.2013, coll. Liu Shuxian and Li Xinxin; $7{ }^{\lambda} 1+$, Tengchong, Zhengding, 1833 m, 6-7.VIII.2013, coll. Liu Shuxian and Li Xinxin; 2 \%, Tengchong, Heinitang, 1824 m, 26-27.VI.2014, coll. Li Xinxin and Pan Xiaodan; 19, Cheli, 620 m, 18.IV.1957, coll. Zang Lingchao; 20̂, Yuanyang, Nansha, 1100 m, 26.V.1979, 
coll. Luo Kezhong; $1{ }^{\Uparrow} 1$ q , Lushui, Liuku, 860-1220 m, 18-19.IX.2014, coll. Liang Hongbin; 2خ4우 Lushui, Pianma, 1750-1980 m, 7.V.1981, 8-12.V.2011, 3-4.VII.2014, coll. Zhang Xuezhong et al.; 1 , Jinping, Mengla, 500 m, 2.V.1956, coll. Huang Keren; 1q, Jinping, Chang Potou, 1200 m, 23.V.1956, coll. Huang Keren. Vietnam (IZCAS): 1ㅇ, Tonkin, Hoa-Binh, leg. A. de Cooman.

Variation. The submarginal line of the forewing varies from dot-like and wavy to faint, broad and interrupted between veins. In the male genitalia, the terminal half of the costa vary from smooth (Fig. 27, IOZ LEP M 01129) to strongly protruding (Fig. 28, IOZ LEP M 08961) among the material on the same region.

Genetic data. The distance to the nearest neighbour C. rectificata is $8.92 \%$. The intrasubspecific divergence of the barcode region of $C$. substigmaria substigmaria ranges from $0 \%-2.6 \%$ (average distance 1\%) $(\mathrm{n}=19)$. Some specimens from Yunnan cluster together at some distance from all other specimens (Fig. 58). Despite the high divergence, no morphological characters were found which separate these populations.

Remarks. After examining the types of $C$. substigmaria brunna, C. tetraspota and a long series of material collected near their type localities, it was found that the external and genital features of $C$. substigmaria brunna and C. tetraspota are nearly identical to those of $C$. substigmaria substigmaria. Barcodes of one paratype of C. tetraspota (IOZ LEP M 02790) and two specimens from type locality of C. substigmaria brunna (IOZ LEP M 17993 and 17994) were clustered within C. substigmaria substigmaria in the Neighbour Joining (NJ) tree with the genetic distances from $0.015 \%-2.6 \%$ (see fig. 58). Thus, C. tetraspota and C. substigmaria brunna are considered as junior synonyms of $C$. substigmaria substigmaria.

Distribution. China (Henan, Shaanxi, Gansu, Jiangsu, Anhui, Zhejiang, Hubei, Jiangxi, Hunan, Fujian, Taiwan, Guangdong, Hainan, Hong Kong, Guangxi, Sichuan, Guizhou, Yunnan), Vietnam.

\section{Cyclidia substigmaria intermedia Prout, 1918}

Figs 9, 29, 40, 51

Cyclidia substigmaria intermedia Prout, 1918:416. Holotype $\widehat{\overbrace{}}$, China: Tibet (BMNH).

Diagnosis. See under C. substigmaria substigmaria.

Type material examined. CHINA: Tibet (BMNH): $1 \overbrace{}^{\lambda}$ (Holotype), Tibet, collector and collecting date unknown, ex. Joicey Collection.

Additional material examined. CHINA: Tibet (IZCAS): 10, Mêdog, Yarang, 1091 m, 20-23.VIII.2006, coll. Lang Songyun; 1 , Mêdog, Beibung, 850 m, 24.VI.1983, coll. Han Yinheng; 2q, Mêdog, 2750 m, 22.VIII.1982, coll. Han Yinheng; 19, Zayü, Dongyan, 1600 m, 17.VII.1973.

Genetic data. No genetic data available.

Distribution. China (Tibet). 


\section{Cyclidia rectificata (Walker, 1862)}

Nelcynda rectificata Walker, 1862a: 1142. Holotype 10, India: Sikkim (BMNH). Cyclidia muricolaria Walker, 1862b: 1483. Holotype 1, India: Darjeeling (BMNH). Cyclidia patulata Walker, 1866: 1537. Holotype , India: Darjeeling (BMNH). Chorodna rectificata: Cotes and Swinhoe 1888: 475.

Enchera rectificata: Hampson 1893: 328.

Cyclidia rectificata: Warren 1922: 445.

Diagnosis. The species is very similar to $C$. diehli Lutz \& Kobes, 2002 (Sumatra) externally, but can be distinguished by the blackish brown and more distinct forewing submarginal line. The most distinct differences are in the male genitalia: the terminal part of the uncus is much narrower and longer; a rounded process with short setae is absent on the basal part of each socius, while $C$. diehli has this character; the terminal part of the valva is much broader than that of $C$. diehli. The male and female genitalia are also similar to those of $C$. substigmaria, the diagnosis can be seen under C. substigmaria substigmaria.

Remarks. There are two subspecies of $C$. rectificata. C. rectificata rectificata (Walker, 1862) is distributed in China and India, and C. rectificata malaisei Bryk, 1943 is distributed in Myanmar.

Distribution. China, India, Myanmar.

\section{Cyclidia rectificata rectificata (Walker, 1862)}

Figs 10, 11, 30, 41, 52

Diagnosis. See under C. rectificata.

Material examined. CHINA: Yunnan (IZCAS): $1 \hat{\jmath}$, Tengchong, Heinitang, 1930 m, 28-30.V.1992, coll. Xue Dayong. Tibet (IZCAS): 79, Nyalam, Zham, 2250 m, 12-20.V.1974, coll. Zhang Xuezhong; 10, Cona, 2800 m, 8.VIII.1974, coll. Huang Fusheng; 1ð, Zham, 2200 m, 25.VI.1975, coll. Wang Ziqing; 1우, Gyirong, 2800 m, 26.VIII.1975, coll. Wang Ziqing; 3우, Bomi, Yi'ong, $2300 \mathrm{~m}$, 23-29.VIII.1983, coll. Han Yinheng; 2ð5 29-31.VIII.2005, coll. Wang Xuejian; 3 ${ }^{\Uparrow} 1$ \% , Nyingchi, Pêlung, 2115 m, 1-2. IX.2005, coll. Wang Xuejian; 4ภ3우, Zayü, Shang Zayü, 1812-1960 m, 21-23.

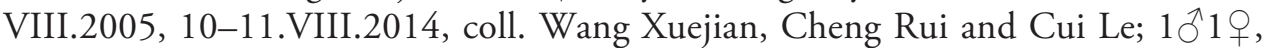
Zayü, Rongcheng Binguan, 2178 m, 8-12.VIII.2014, coll. Cheng Rui and Cui Le; 2ㅇ, Mainling, Pai, 2883 m, 4-6.VIII.2006, coll. Lang Songyun; 8ô11우, Mêdog, Lage, 3213 m, 7-8.VIII.2006, coll. Lang Songyun; $3{ }^{\Uparrow} 2$, Mêdog, Dayandong, 2880 m, 9.VIII.2006, coll. Lang Songyun; 20̂, Mêdog, Hanmi, 2095 m, 10-11. VIII.2006, coll. Lang Songyun; 2ㅇ, Mêdog, Pomo Gonglu 80K, 2118 m, 24-25. VIII.2006, coll. Lang Songyun. 
Genetic data. The intraspecific divergence of the barcode region of $C$. rectificata rectificata is $0 \%$ (average distance $0 \%)(\mathrm{n}=7)$. The distance to the nearest neighbour C. substigmaria is $8.92 \%$.

Distribution. China (Yunnan, Tibet), India.

\section{Cyclidia pitimani (Moore, 1886)}

Figs 12, 13, 31, 42

Euchera pitimani Moore, 1886: 99. Syntypes including 1 $\overbrace{}^{\lambda}$, Burma: Tenasserim, Tavoy (BMNH).

Cyclidia pitimani: Warren, 1922: 445.

Cyclidia sericea Warren sensu Chu \& Wang, 1987: 206. (Misidentification)

Cyclidia sericea Warren sensu Chu \& Wang, 1991: 64, fig. 24, pl. 1: 4. (Misidentification)

Diagnosis. This species is very similar to $C$. sericea (Borneo, Sumatra), but can be distinguished by the following characters: smaller than $C$. sericea (the average forewing length of the male is $32 \mathrm{~mm}$, against $c a 40 \mathrm{~mm}$ in C. sericea); in C. pitimani, the doubled antemedial line form almost right angles anteriorly, especially the inner line, while in $C$. sericea, the protrusions of the antemedial lines are more rounded; the anterior part of the median band is much narrower in C. pitimani; the terminal spots are less distinct than those of $C$. sericea. In the male genitalia, the terminal part of the valva is broader and more rounded.

Material examined. CHINA: Yunnan (IZCAS): $2 \hat{\jmath}$, Xishuangbanna, Xiaomengyang, $850 \mathrm{~m}, 6-7 . I X .1957$, coll. Zang Lingchao and Zhang Yiran (one male was originally incorrectly recorded as "Qinghai, Gonghe"); 2ภ, Xishuangbanna, Bubang, 700 m, 14.IX.1993, coll. Yang Longlong.

Genetic data. No genetic data available.

Remarks. After examining the types of $C$. pitimani and C. sericea, and studying the descriptions and figures of the two species (Moore 1886, Warren 1922, Holloway 1998, Chen 2011), we found that the specimens from Yunnan which were identified as C. sericea by Chu and Wang $(1987,1991)$ well agree with C. pitimani. Thus, C. sericea in Chu and Wang $(1987,1991)$ is considered to be a misidentification of C. pitimani.

Chu and Wang $(1987,1991)$ recorded one male specimen from "Qinghai, Gonghe, $3150 \mathrm{~m}, 6$. IX.1957, coll. Zang Lingchao". After examination, it was noted that the locality on the label of this specimen was incorrect. According to the collecting records of IZCAS, the collector (Zang Lingchao) went to Xiaomengyang of Xishuangbanna in Yunnan on September 6th, 1957, and no collector went to Qinghai on that date. We also found another specimen of $C$. pitimani which was collected at the same locality on September 7th, 1957. So, the locality on label should be written as Yunnan, Xishuangbanna, Xiaomengyang, $850 \mathrm{~m}$. Qinghai should be deleted from the range area of C. sericed and the species should be deleted from the fauna of China.

Distribution. China (Yunnan), Myanmar. 


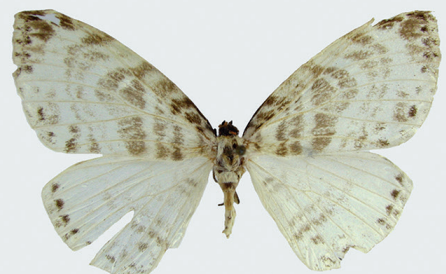

12

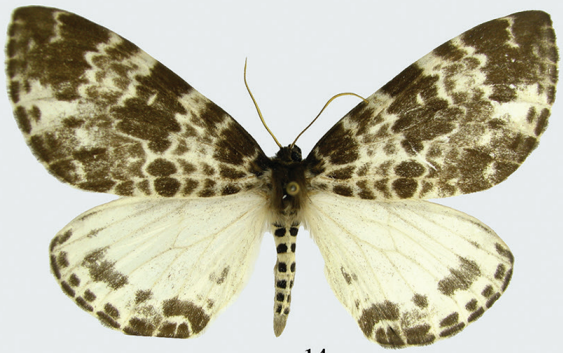

14

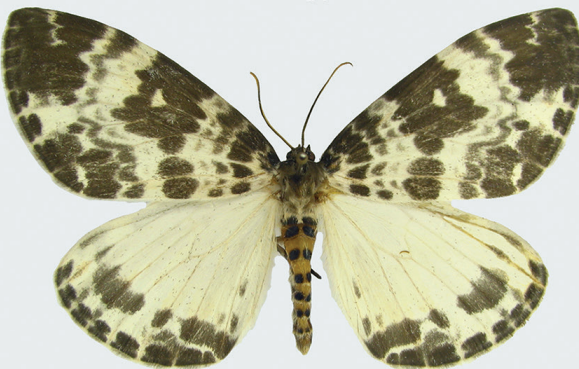

16
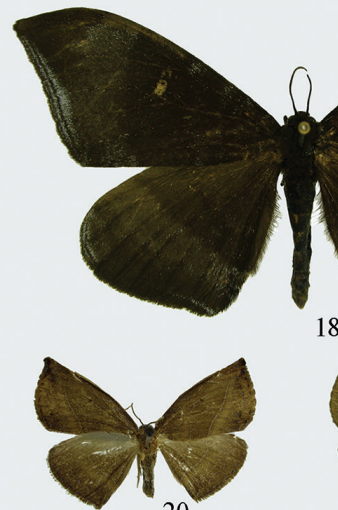

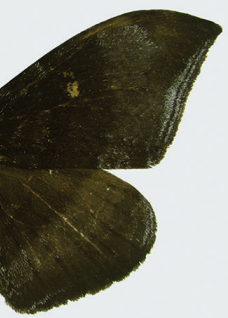

18

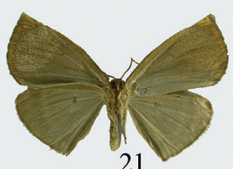

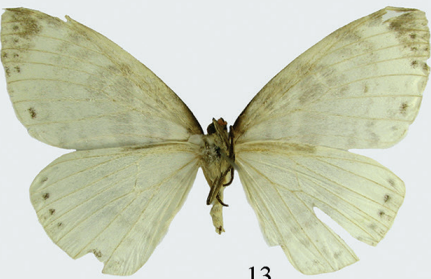

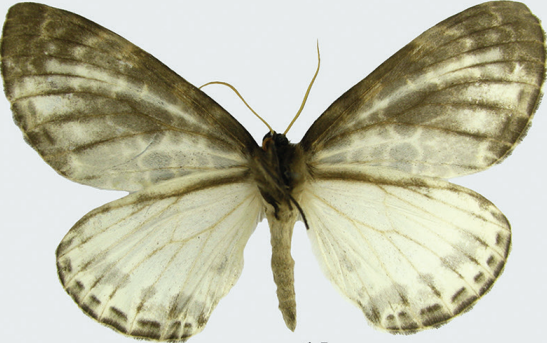

15

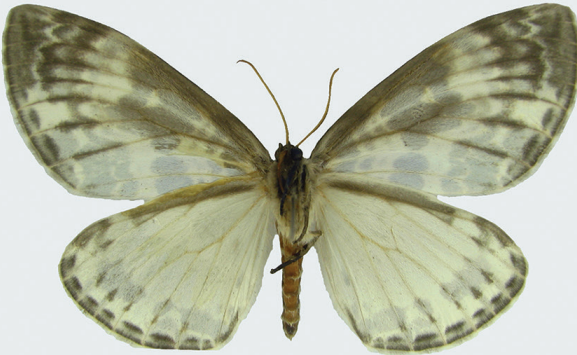

17

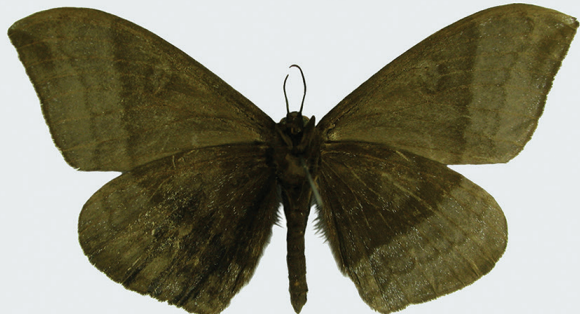

19

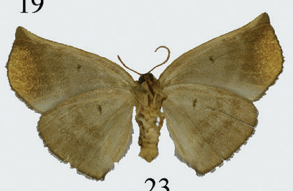

Figures I 2-23. Adults. I 2-I 3 Cyclidia pitimani I 2 male (Yunnan) I 3 ditto, underside I 4-I 5 C. fractifasciata fractifasciata $\mathbf{1 4}$ male (Yunnan) $\mathbf{1 5}$ ditto, underside 16-17 C. fractifasciata indistincta subsp. n. 16 male (holotype, Gansu) 17 ditto, underside 18 -19 C. orciferaria 18 male (Hainan) 19 ditto, underside 20-21 Mimozethes angula 20 male (holotype, Sichuan) $\mathbf{2}$ ditto, underside 22-23 M. lilacinaria 22 male (holotype, Sichuan) 23 ditto, underside. Scale bar: $1 \mathrm{~cm}$. 

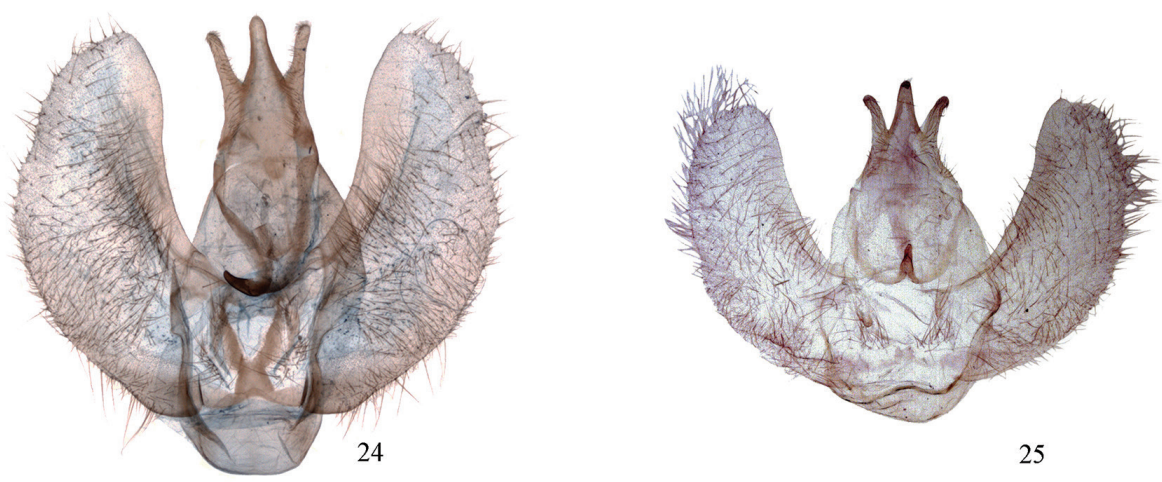

25
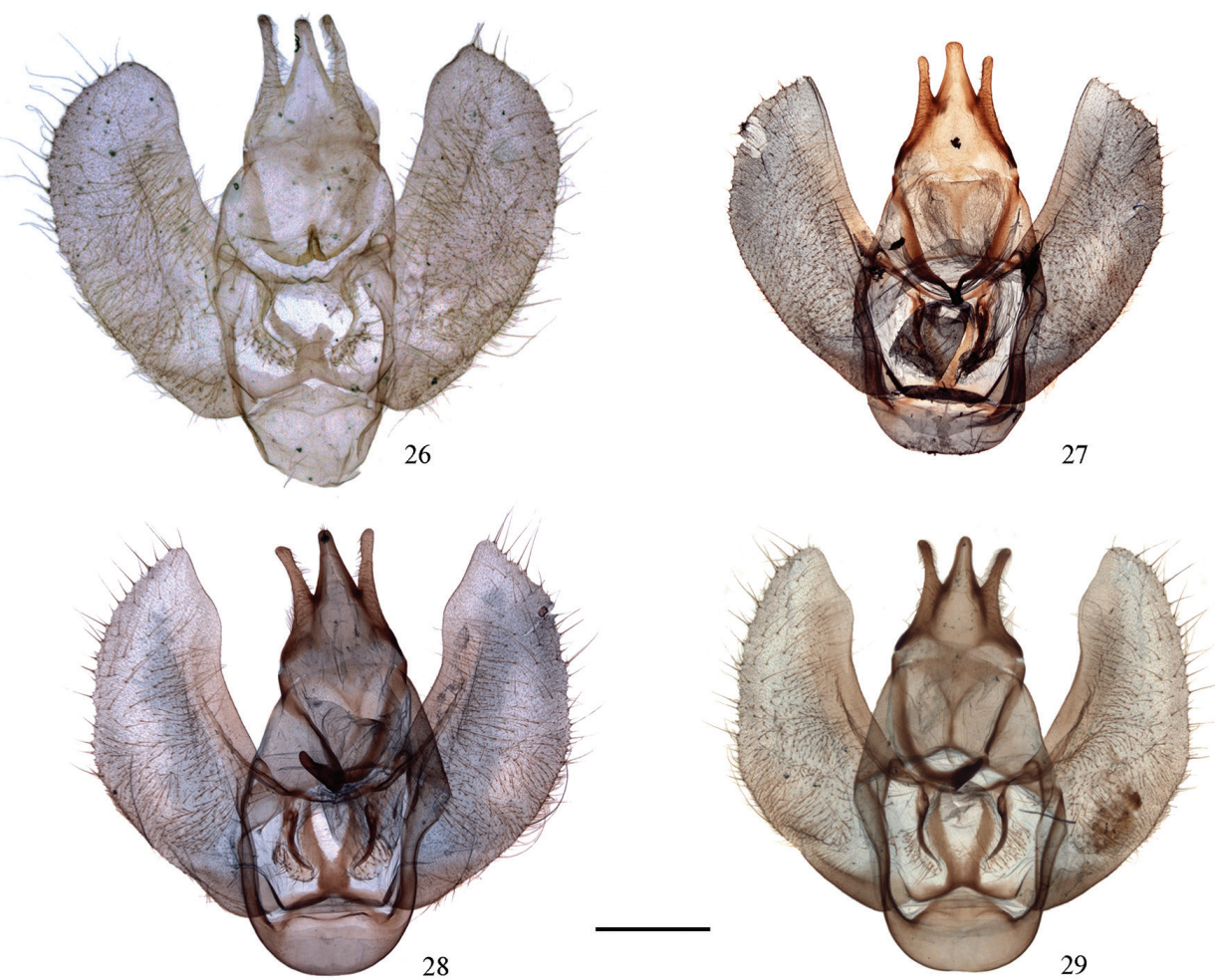

Figures 24-29. Male genitalia of Cyclidia. 24 C. substigmaria substigmaria (Baoshan, Yunnan, slide no. 41) $\mathbf{2 5}$ ditto (holotype of C. substigmaria brunna, Emeishan, Sichuan, slide no. 12) 26 ditto (holotype of C. tetraspota, Xishuangbanna, Yunnan, slide no. 10) 27 ditto (Xishuangbanna, Yunnan, slide no. 681) 28 ditto (Xishuangbanna, Yunnan, slide no. 683) 29 C. substigmaria intermedia (Tibet, slide no. 311). Scale bar: $1 \mathrm{~mm}$. 


\section{Cyclidia fractifasciata (Leech, 1898)}

Euchera fractifasciata Leech, 1898: 360. Syntypes 10ึ, 1, China: Western China (BMNH).

Cyclidia fractifasciata: Gaede 1931: 2.

Diagnosis. The species can be distinguished by the following characters: a black broad subbasal line is present on the forewing; the forewing medial line is broad at anterior half and very narrow and dot-like at posterior half; outer margin of the forewing medial line forms an almost right angle below $\mathrm{M}_{3}$; the phallus of the male genitalia forms a small protrusion posteriorly; the lamella postvaginalis of the female genitalia is rectangle.

Remarks. Chu and Wang (1991) did not record this species. The specimens from Yunnan should be identified as $C$. fractifasciata fractifasciata, and the specimens from Gansu and Chongqing should be identified as a new subspecies, $C$. fractifasciata indistincta subsp. n., based on adult morphology and DNA barcodes.

Distribution. China.

\section{Cyclidia fractifasciata fractifasciata (Leech, 1898)}

Figs 14, 15, 32, 43, 53

Diagnosis. See under C. fractifasciata indistincta.

Material examined. CHINA: Yunnan (IZCAS): 10, Dulongjiang, $1500 \mathrm{~m}$, 29.V.2006, coll. Xiao Ningnian; 3ठำ, Lushui, Pianma, 8-12.V.2011, coll. Yang Xiushuai and Wang Ke.

Distribution. China (Yunnan).

Genetic data. The intrasubspecific divergence of the barcode region in C. fractifasciata fractifasciata is $0 \%(\mathrm{n}=4)$.

\section{Cyclidia fractifasciata indistincta Jiang, Han \& Xue, subsp. n.} http://zoobank.org/8BC2D3BA-389A-43E9-991E-CA93B3DE1837

Figs $16,17,33,44,54$

Description. Head. Antennae blackish brown dorsally, flat and unipectinate, basal half without rami, rami very short. Frons blackish grey, not protruding. Labial palpi black with third segment distinct, extending beyond frons. Vertex black scattered with grey scales.

Thorax. Patagia white at basal half and blackish grey at terminal half. Tegula blackish grey. Dorsal side of thorax white with two pairs of blackish grey patches medially. 
Hind tibia with two pairs of spurs in both sexes. Forewing length: $37-40 \mathrm{~mm}$. Apex of forewing rounded, not falcate; outer margin of both wings smooth. Wings white, transverse lines black. Forewing with a blackish brown patch basally; subbasal line broad; antemedial lines double, outer line indistinct and often invisible; medial line broad band-like at anterior half, very narrow and dot-like at posterior half; outer margin of medial line forming an almost right angle below $\mathrm{M}_{3}$; discal spot white, almost rhombic; postmedial lines double, wavy, inner line very obscure; submarginal line double, broad, and invisible between $\mathrm{M}_{3}$ and $\mathrm{CuA}_{1}$; terminal lines double and discontinuous on each vein, inner line composed of oval markings, outer line appearing as series of short strips, inner markings often fused with outer ones; fringes white mixed with blackish grey. Hind wing with indistinct submarginal line; terminal lines and fringes similar to those of forewing. Underside white, striations indistinct than those of upperside.

Abdomen. Abdominal segments diffused with white scales. Pairs of black quadrate markings on first to seventh abdominal segments. Anterotergal syndeses developed at anterior margin of $2^{\text {nd }}$ tergum. A pair of androconial hair-pencils present on $2^{\text {nd }}$ pleuron of male.

Male genitalia. Uncus triangular. Socii sclerotized, about four-fifths the length of uncus. Gnathos with median process small and triangular. Valva narrow terminally; costa sclerotized and almost straight. Juxta formed a pair of forcipiform processes posteriorly. Saccus semicircular, about two-fifths length of basal width. Phallus slightly curved, with a small triangular lateral process posteriorly; vesica without cornuti.

Female genitalia. Lamella postvaginalis rectangle. Ductus bursae with a colliculum, long and narrow, striate longitudinally. Corpus bursae oval, with a paired slender signa; signa separated and parallel.

Diagnosis. The subspecies is very similar to the nominate subspecies, but differs externally by the following characters: the outer line of the antemedial line and the inner line of the postmedial line on the forewing are invisible, while in the nominate subspecies, they are much more distinct; the forewing discal spot is larger; the inner terminal markings of the forewing are larger and fused with the outer ones partly, while in $C$. fractifasciata fractifasciata, they are often smaller and separated from the outer ones.

Type material examined. Holotype, $\widehat{\sigma}$, CHINA: Gansu (IZCAS): Kangxian, Yangba, Meiyuangou, 1000 m, 13.VIII.2014, coll. Xue Dayong and Ban Xiaoshuang. Paratypes: $3 \AA 2$, same data as holotype. Chongqing (IZCAS): 1 , W Wushan, Wulipo, Dangyang, Congping, 1773 m, 25.VII.2013, coll. Cheng Rui.

Genetic data. The intrasubspecific divergence of the barcode region in C. fractifasciata indistincta is $1 \%$. The intraspecific divergence of the barcode region between $C$. fractifasciata fractifasciata $(\mathrm{n}=4)$ and C. fractifasciata indistincta $(\mathrm{n}=5)$ is $2.3 \%$. The distance between $C$. fractifasciata with the nearest neighbour species C. substigmaria is $12.5 \%$.

Distribution. China (Gansu, Chongqing).

Etymology. The subspecies is named on the basis of the Latin adjective indistinctus, referring to the transverse lines of the forewing. 

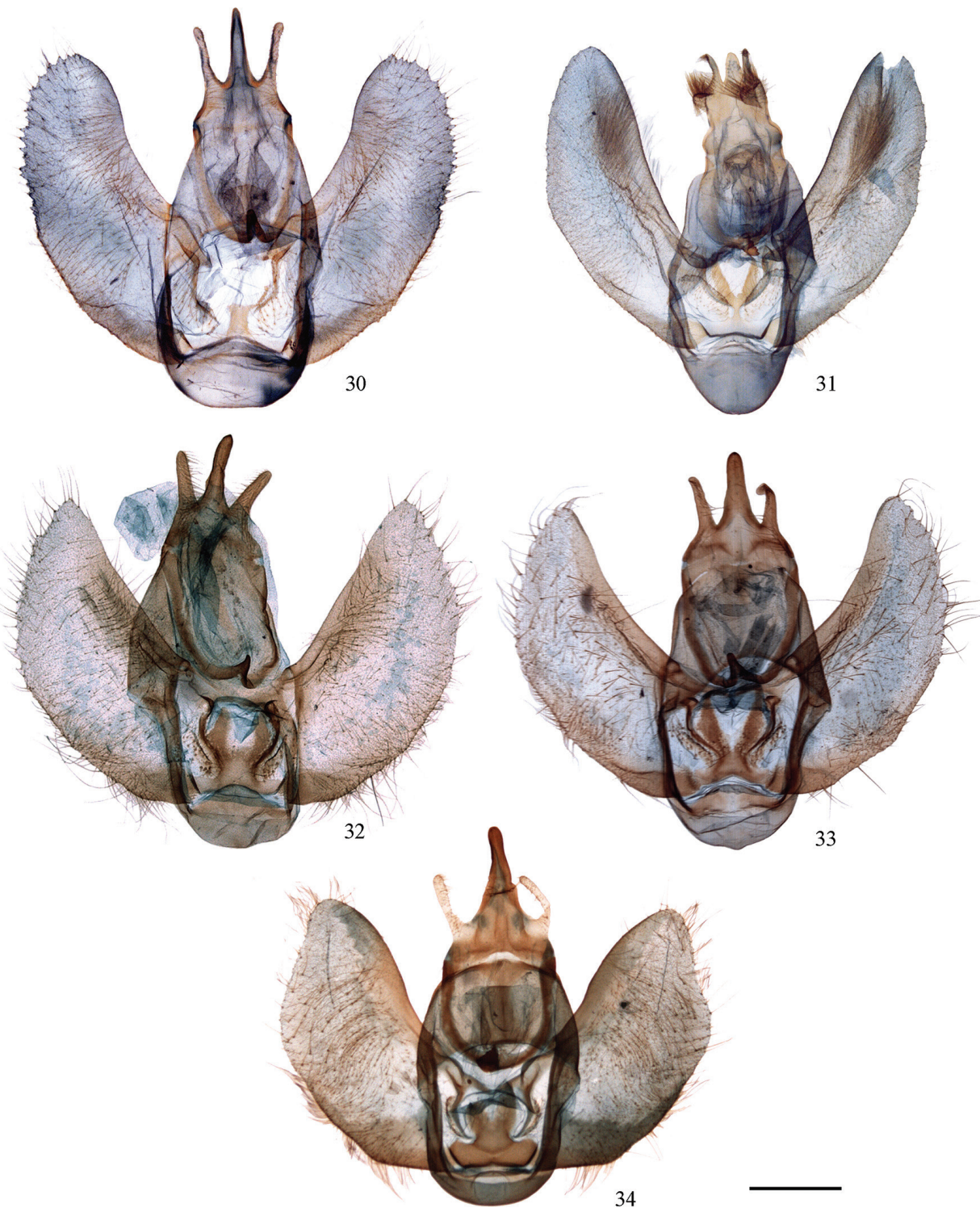

Figures 30-34. Male genitalia of Cyclidia. 30 C. rectificata (Tibet, slide no. 2) 3I C. pitimani (Yunnan, slide no. 9) 32 C. fractifasciata fractifasciata (Yunnan, slide no. 724) 33 C. fractifasciata indistincta subsp. n. (paratype, Gansu, slide no. 721) 34 C. orciferaria (Hainan, slide no. 728). Scale bar: $1 \mathrm{~mm}$. 

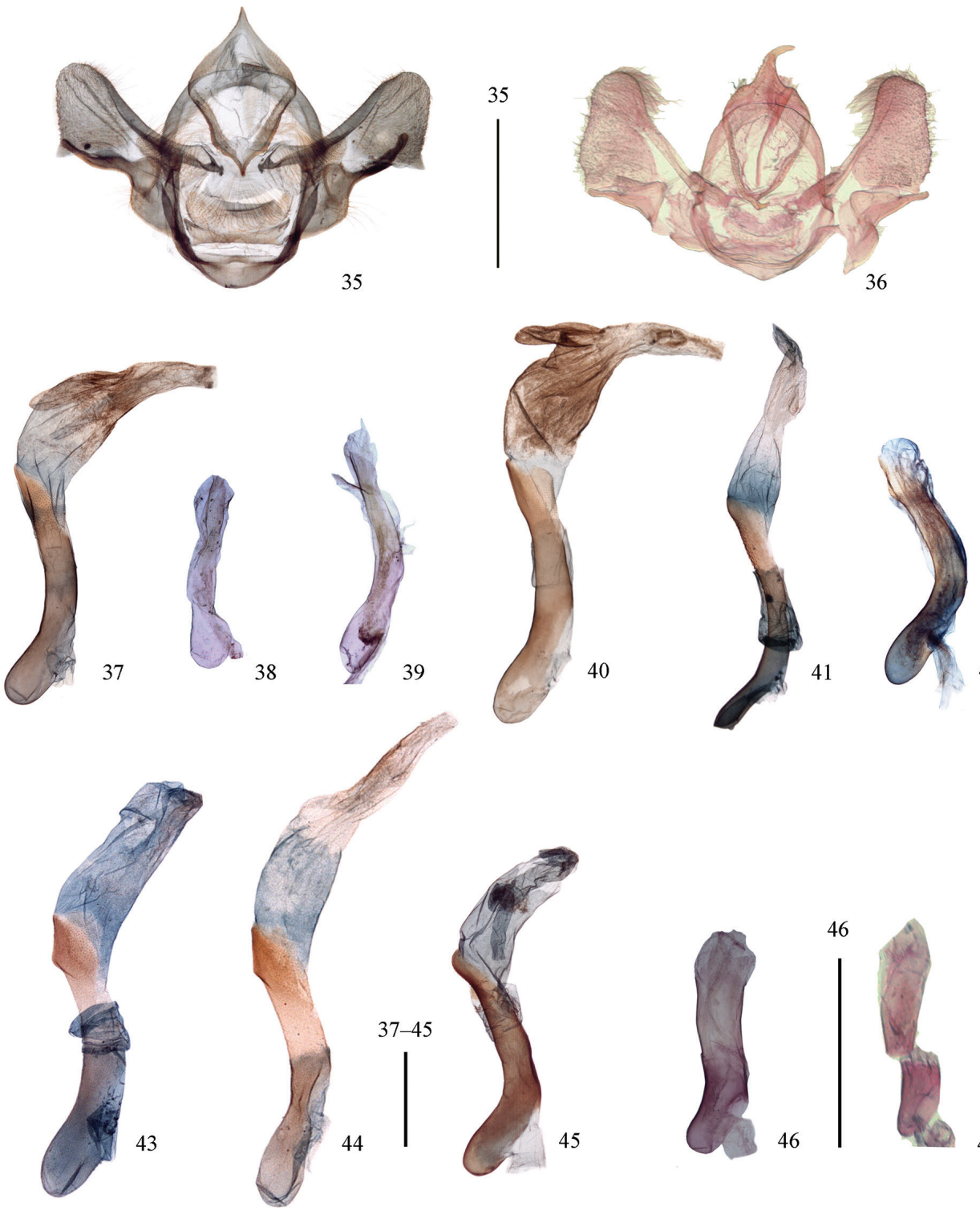

Figures 35-47. 35-36 Male genitalia of Mimozethes. 35 M. angula (paratype, Sichuan, slide no. 34) $36 \mathrm{M}$. lilacinaria (Sichuan, BMNH, slide No. 304) 37-47 Phallus 37 Cyclidia substigmaria substigmaria (Tengchong, Yunnan, slide no. 682) 38 ditto (holotype of C. substigmaria brunna, Emeishan, Sichuan, slide no. 12) 39 ditto (holotype of C. tetraspota, Xishuangbanna, Yunnan, slide no. 10) $\mathbf{4 0}$ C. substigmaria intermedia (Tibet, slide no. 311) 4 I C. rectificata (Tibet, slide no. 727) 42 C. pitimani (Yunnan, slide no. 9) 43 C. fractifasciata fractifasciata (Yunnan, slide no. 724) 44 C. fractifasciata indistincta subsp. n. (paratype, Gansu, slide no. 721) 45 C. orciferaria (Hainan, slide no. 728) 46 M. angula (holotype, Sichuan, slide no. 19) 47 M. lilacinaria (Sichuan, BMNH, slide No. 304). Scale bars: $1 \mathrm{~mm}$. 


\section{Cyclidia orciferaria Walker, 1860}

Figs 18, 19, 34, 45, 55

Cyclidia orciferaria Walker, 1860: 56. Syntypes, China: North China.

Cyclidia ociferaria Kirby, 1892: 725. [Incorrect spelling of Cyclidia orciferaria Walker.]

Diagnosis. This species is different from other congeners in the following external characters: the apex of the forewing is falcate; the wing colour is blackish brown; two bands covered with greyish blue scales are present on the forewing, and the inner band is narrower and less distinct than the outer band; the discal spot of the forewing is yellowish brown, oblong, with a blackish brown narrow line medially; greyish blue scales are covered on the submarginal lines of both wings, and often absent on the middle part of the hind wing. There are also differences in the male genitalia: the socii are weakly sclerotized and much shorter than the uncus; the valva is short. In the female genitalia, the posterior margin of the lamella postvaginalis is slightly concaved; the two signa are tapered at posterior half and situated very close to each other.

Material examined. CHINA: Zhejiang (IZCAS): 20, Tianmushan, 20-23. VII.1973, coll. Zhang Baolin; 1ô1우, Lin'an, West Tianmushan, 400-1500 m, 26.VII-29.VIII.2003, coll. Xue Dayong et al.; 1ð̄, West Tianmushan, Zhonglieci, 363 m, 24.VII.2011, coll. Yan Keji; 10̄, West Tianmushan, Xianrending, 1506 m, 27.VII.2011, coll. Yan Keji; $2 \hat{\jmath} 2$, Taishun, Wuyanling, Shuangkengkou, 680 m, 28-29.VII.2005, coll. Lang Songyun; 1 9 , Taishun, Siqianzhen, 250 m, 4.VIII.2005,

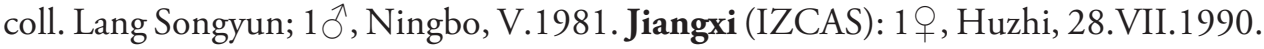
Hunan (IZCAS): $2{ }^{\lambda}$, Yanling, Taoyuandong, $631 \mathrm{~m}$, 4-8.VII.2008, coll. Chen Fu-

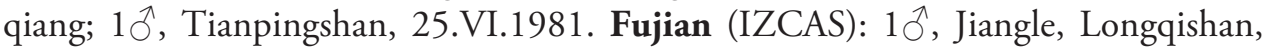
800 m, 15.IX.1990, coll. Yang Bin; 80, Wuyishan, 24.IV-21.V.1983, coll. Wang Linyao; 1 , W Wyishan, Sangang, 24.VII.1980; 1옹 Nanping, Shangyang, 9.VI.1963, coll. Zhang Youwei. Guangdong (IZCAS): $1{ }^{\lambda}$, Ruyuan, Nanling, Baohuzhan, 1020 m, 16-20.VII.2008, coll. Chen Fuqiang; 1 9 , Shixing, Chebaling, 365-401 m, 22-26. VII.2008, coll. Chen Fuqiang. Hainan (IZCAS): $4 \overbrace{}^{\Uparrow} 2$, Nankai, Nanmaola, 1261 m, 10-14.V.2009, coll. Chen Fuqiang and Yan Keji; 6 1 엉, Jianfengling, Tianchi, 828 m, 1-5.V.2007, 18.V.2009, coll. Chen Fuqiang; 1 2 2 , Bawangling, Dong'er Linchang, 1004-1015 m, 8.V.2007, 7.IV.2008, coll. Chen Fuqiang and Lang Songyun; 11 $\hat{\text {, }}$, Wuzhishan, Shuiman, 730-900 m, 7-11.V.2007, 1-3.IV.2008, coll. Lang Songyun and Han Hongxiang; 1 33 ㅇ, Lingshui, Diaoluoshan, 190-920 m, 3-7.V.2007, coll. Han Hongxiang and Lang Songyun; 19, Qiongzhong, Limuling, 620 m, 15.V.2007, coll. Han Hongxiang; 19, Xinglong, 24.IV.1963, coll. Zhang Baolin. Guangxi (IZCAS): 3 2 2 , Fangcheng, Fulong, 200-550 m, 23-26.V.1999,

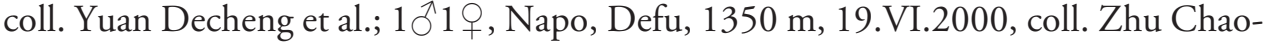

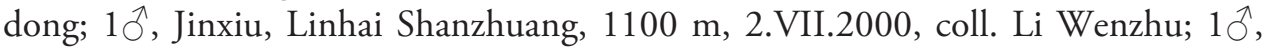
Jinxiu, Jinzhong Gonglu, 1000 m, 10.V.1999, coll. Han Hongxiang; 1올 Daxin, Xialei, 680 m, 31.III.1998, coll. Li Wenzhu. Yunnan (IZCAS): 101 19, Hekou, Xiaonanxi, 200 m, 10-11.VI.1956, coll. Huang Keren et al.; 1 9 , Pingbian, Daweis- 


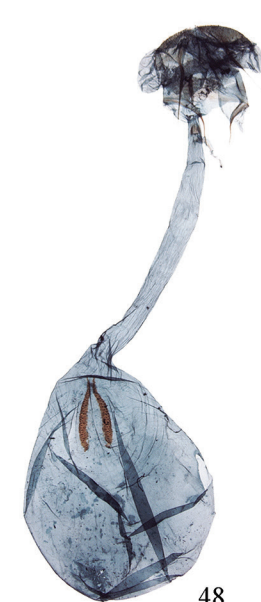

48

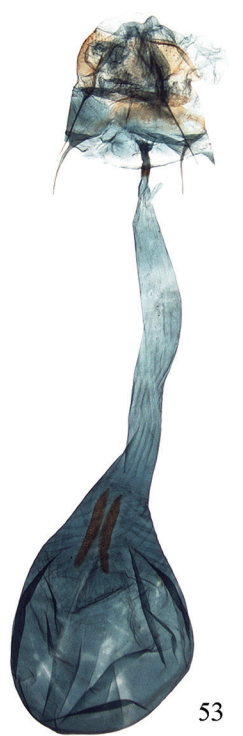

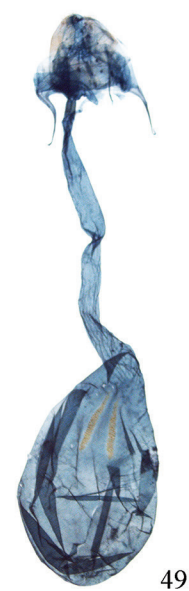

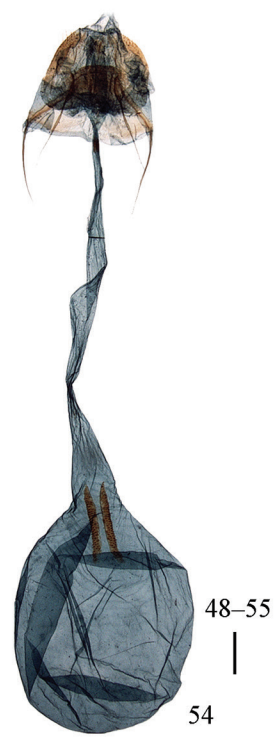

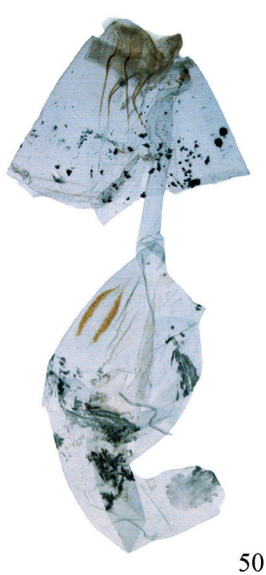
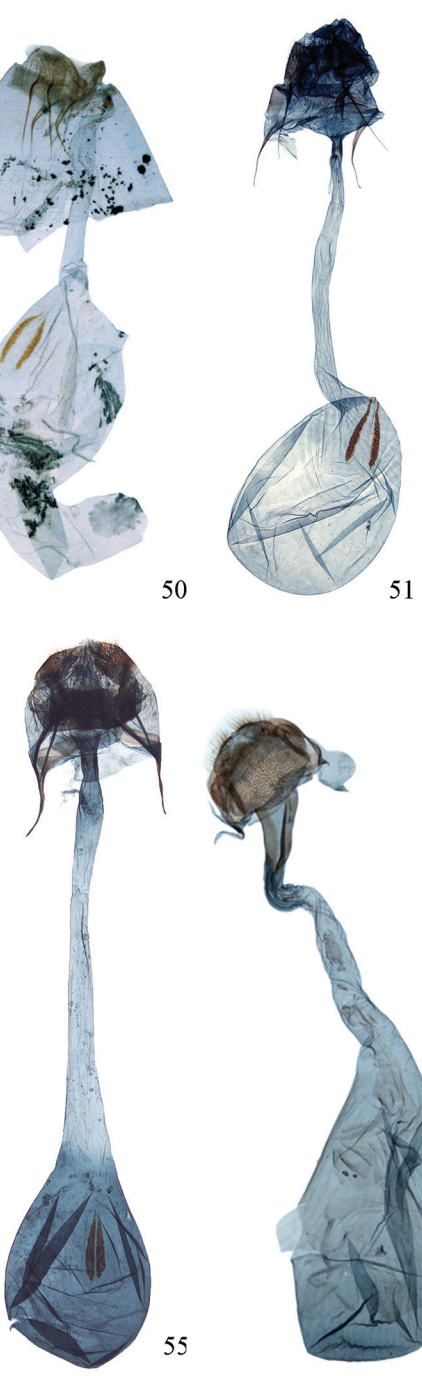

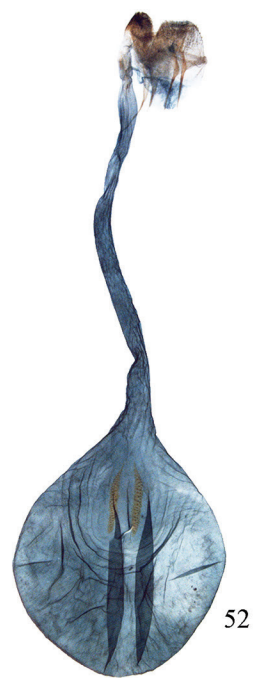

Figures 48-57. Female genitalia. 48 Cyclidia substigmaria substigmaria (Henan, sldie. no. 726$) 49$ ditto (Jiangsu, slide. no. 33) $\mathbf{5 0}$ ditto (paratype of C. tetraspota, Xishuangbanna, Yunnan, slide no. 36) 5 I C. substigmaria intermedia (Tibet, slide no. 685) $\mathbf{5 2}$ C. rectificata (Tibet, slide no. 3) $\mathbf{5 3}$ C. fractifasciata fractifasciata (Yunnan, slide no. 725) 54 C. fractifasciata indistincta subsp. n. (paratype, Gansu, slide no. 722) 55 C. orciferaria (Hainan, slide no. 729) 56 M. angula (Henan, slide no. 288) 57 M. lilacinaria (Sichuan, slide no. 280). Scale bars: $1 \mathrm{~mm}$.

han, 1500 m, 20.VI.1956, coll. Huang Keren et al.; 10^, Xishuangbanna, Mengla, Menglun, $650 \mathrm{~m}, 1$. VI.1964, coll. Zhang Baolin; $1{ }^{\lambda} 1$, , Mengla Linchang, $550 \mathrm{~m}$, 20.IV.1982, coll. Wang Yongxian; 10, Mengla, 20.VI.1982, coll. Chen Yixin; 19, Mengla, Lengku, 623 m, 10.VI.1980, coll. Guo Zuyun; 10̂, Xishuangbanna, Bubang, 700 m, 14.IX.1993, coll. Yang Longlong; 1 3 , Xishuangbanna, Damenglong, 650 
m, 1.VIII.1958, coll. Zheng Leyi; 10, Xishuangbanna, Dameng'a, 1050-1080m, 15.VIII.1958, coll. Wang Shuyong; 20, Cangyuan, 790-1100 m, 19-22.V.1980, coll. Song Shimei and Shang Jinwen; $1 \delta^{\Uparrow}$, Xiaomenglun, 21.IV.1982, coll. Wang Lin-

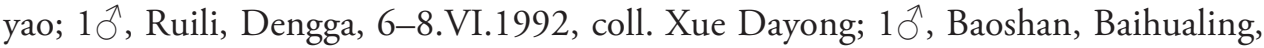
1520 m, 11-13.VIII.2007, coll. Wu Chunguang.

Genetic data. The intraspecific divergence of the barcode region of $C$. orciferaria is ranges from $0 \%-1.7 \%$ (average distance $1.09 \%)(\mathrm{n}=8)$. The distance to the nearest neighbour C. substigmaria is $11.5 \%$.

Distribution. China (Jiangsu, Zhejiang, Jiangxi, Hunan, Fujian, Guangdong, Hainan, Guangxi, Sichuan, Yunnan), Myanmar, Vietnam, Indonesia.

Biological notes. The morphology of the larva of $C$. orciferaria was illustrated in Chen (2011).

\section{Mimozethes Warren, 1901}

Mimozethes Warren, 1901: 190. Type species: Euchera nana Warren, 1897, by original designation.

Generic characters. Head. Antennae lamellate and shortly unipectinate, basal part of antennae without rami (Fig. 1b). Frons not protruding. Labial palpi with second segment slightly curved, third segment oval. Thorax. Hind tibia with two pairs of spurs. Apex of forewing falcate; outer margin of forewing protruding. Wing. Wings colour dark brown. Forewing with silver grey antemedial line, sometimes indistinct; discal spot black and small; postmedial line silver grey, forming a ">" shaped protrusion near $\mathrm{R}_{5}$. Hind wing with medial line and postmedial line silver grey and almost straight. Black brown patches present near anal angle of both wings. Terminal lines of both wings composed of a series of blackish brown strips covering silver grey scales, very distinct towards apex. Underside with distinct discal spot, costa, apex and outer margin suffused with pale yellowish brown scales. Vein (Fig. 3b). Forewing with $\mathrm{R}_{1}$ separate, $\mathrm{R}_{2-4}$ and $\mathrm{R}_{5}$ stalked, $\mathrm{M}_{2}$ arising from middle of discocellulars; Hind wing with $S_{c}+R_{1}$ close to Rs beyond distal cell, then far from $R s, M_{2}$ arising from middle of discocellulars. Anterotergal syndeses developed at anterior margin of $2^{\text {nd }}$ tergum (Fig. 2). A pair of androconial hair-pencils present on $2^{\text {nd }}$ sternum of male (Fig. 2). Male genitalia. Uncus triangular, acute terminally; socii undeveloped; gnathos connected at middle and with median process small and acute apically; sacculus forming a long process; juxta short and broad, concaved posteriorly; saccus broad and rounded terminally; Phallus short; vesica without cornuti. Female genitalia. Papillae anales broad and rounded; lamella postvaginalis large and oval, with many tiny spines; ductus bursae long and narrow, with a colliculum; corpus bursae oval, without a signum.

Diagnosis. See under Cyclidia. 
Remarks. According to Inoue (1962), M. argentilinearia (Leech, 1897) occurs in Japan and Taiwan. However, it has not been recorded from Taiwan in later studies (Inoue 1992, Yan et al. 2009, Chen 2011). Thus, following that, we do not include the species in this paper.

Distribution. China, Japan.

\section{Key to Chinese Mimozethes species}

$1 \quad$ Outer margin of forewing weakly protruding; ventral margin of valva forming a small triangular protrusion apically in male genitalia.......M. angula, Figs 20-21

- $\quad$ Outer margin of forewing strongly protruding; ventral margin of valva not forming a small triangular protrusion apically in male genitalia

M. lilacinaria, Figs 22-23

\section{Mimozethes angula Chu \& Wang, 1987}

Figs 20, 21, 35, 46, 56

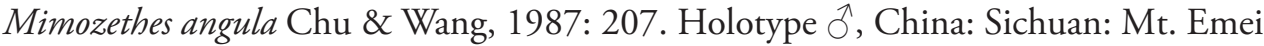
(IZCAS).

Diagnosis. This species is very similar to M. lilacinaria (Leech, 1897) and M. argentilinearia, but it can be distinguished by the following characters: the outer margin of the forewing is less strongly protruding than that of M. lilacinaria and M. argentilinearia; the black patch inside the anal angle of the forewing is less distinct than that of $M$. argentilinearia; the yellowish brown patch on the underside of the forewing is much smaller and less distinct than that of $M$. lilacinaria and $M$. argentilinearia. In the male genitalia, the uncus is shorter; the ventral margin of the valva forms a small triangular protrusion apically, but $M$. lilacinaria and $M$. argentilinearia lack this character; the sacculus process is much longer than that of $M$. lilacinaria.

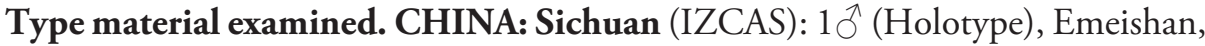
Qingyinge, 800-1000 m, 15.IX.1957, coll. Zhu Fuxing; 1 ㅇ (Allotype), same locality, 22.IX.1957, coll. Zhu Fuxing; 4仓2ㅇ (Paratype), same locality, 22.VI.1957, 15-19. IX.1957, coll. Zhu Fuxing et al.

Additional material examined. CHINA: Henan (IZCAS): 1ㅇ, Baiyunshan, 13-15.VIII.2008, 1550 m, coll. Jiang Nan. Hubei (IZCAS): 10, Shennongjia, Dajiuhu, 1800 m, 1.VIII.1981, coll. Han Yingheng. Sichuan (IZCAS): 9ð2ㅇ, Emeishan, Qingyinge, 800-1000 m, 20.VI.1957, 15-22.IX.1957, coll. Zhu Fuxing et al.; 1웅 Qingchengshan, $1000 \mathrm{~m}, 4 . V I .1979$, coll. Shang Jinwen; 10, Emeishan, $1288 \mathrm{~m}$, 31.VII.2013, coll. Cheng Rui.

Genetic data. No genetic data available.

Distribution. China (Henan, Hubei, Sichuan). 
Mimozethes lilacinaria (Leech, 1897)

Figs 22, 23, 36, 47, 57

Decetia lilacinaria Leech, 1897: 184. Holotype ô, China: Sichuan: Emeishan (BMNH).

Heteromize lycoraearia Oberthür, 1912: 269. Holotype ô, China: Sichuan: Mou-pin (BMNH).

Mimozethes lilacinaria: Beccaloni et al. 2003 [accessed 26 November 2015].

Diagnosis. See under $M$. angula.

Type material examined. CHINA: Sichuan (BMNH): $1 \delta^{\lambda}$ (Holotype), OmeiShan, 3620 ft., Native coll. July \& Aug. 1890, Leech Coll. 1900-64, BMNH (E) 1377104.

Additional material examined. CHINA: Sichuan (BMNH): 10 , Chasseurs indigènes, de Tà-tsien-lou, Récolle de 1910, Ex Oberthür Coll. Brit. Mus. 1927-3, Drepanidae genitalia slide No. 304; 19, Siao-Lou, 1900, Chasseurs indigènes, Ex Oberthür Coll. Brit. Mus. 1927-3. Yunnan (IZCAS): 1 9 , Xishuangbanna, Menghai, 21.VII.1958, coll. Wang Shuyong.

Genetic data. No genetic data available.

Remarks. Chu and Wang (1991) did not record this species. The specimens from Yunnan should be identified as M. lilacinaria based on adult morphology.

Distribution. China (Sichuan, Yunnan).

\section{DNA barcoding results and discussion}

Forty-three DNA barcode sequences of lengths 658bp were obtained for Cyclidia species. The nucleotide composition of Cyclidia species COI genes was $30.60 \%$ of A, 38.54 of $\mathrm{T}, 16.06 \%$ of $\mathrm{C}, 14.80 \%$ of $\mathrm{G}$. The interspecific distance within the genus was range from $8.8 \%-13.9 \%$. The maximum intraspecific distances was $2.6 \%$ in $C$. substigmaria, $1.7 \%$ in $C$. orciferaria, $0.0 \%$ in C. rectificata, and $2.3 \%$ in $C$. fractifasciata. The maximum genetic distances observed within species $(2.6 \%$ at $\mathrm{COI})$ were less than the minimum distances observed between the species $(8.8 \%)$. There is a clear barcoding gap between intra and interspecific variation; furthermore, $\mathrm{NJ}$ tree also provided strong support for the separation of Cyclidia species (Fig. 58).

In recent revisionary work of Drepanidae, Song et al. $(2011,2012)$ and Park et al. (2011) found many new taxa, synonyms and misidentifications in earlier studies. However, when dealing with some morphologically similar taxa, it is difficult to discriminate only using the subtle diagnostic characters. The present study utilizing morphological and molecular characters revised some Chinese Cyclidia species. The morphological analysis indicated that some structures of the genitalia were found to be less diagnostic than the external characters between some species (i.e. C. substigmaria and $C$. rectificata). Sihvonen et al. (2014) also mentioned this trait in the Geometridae. 


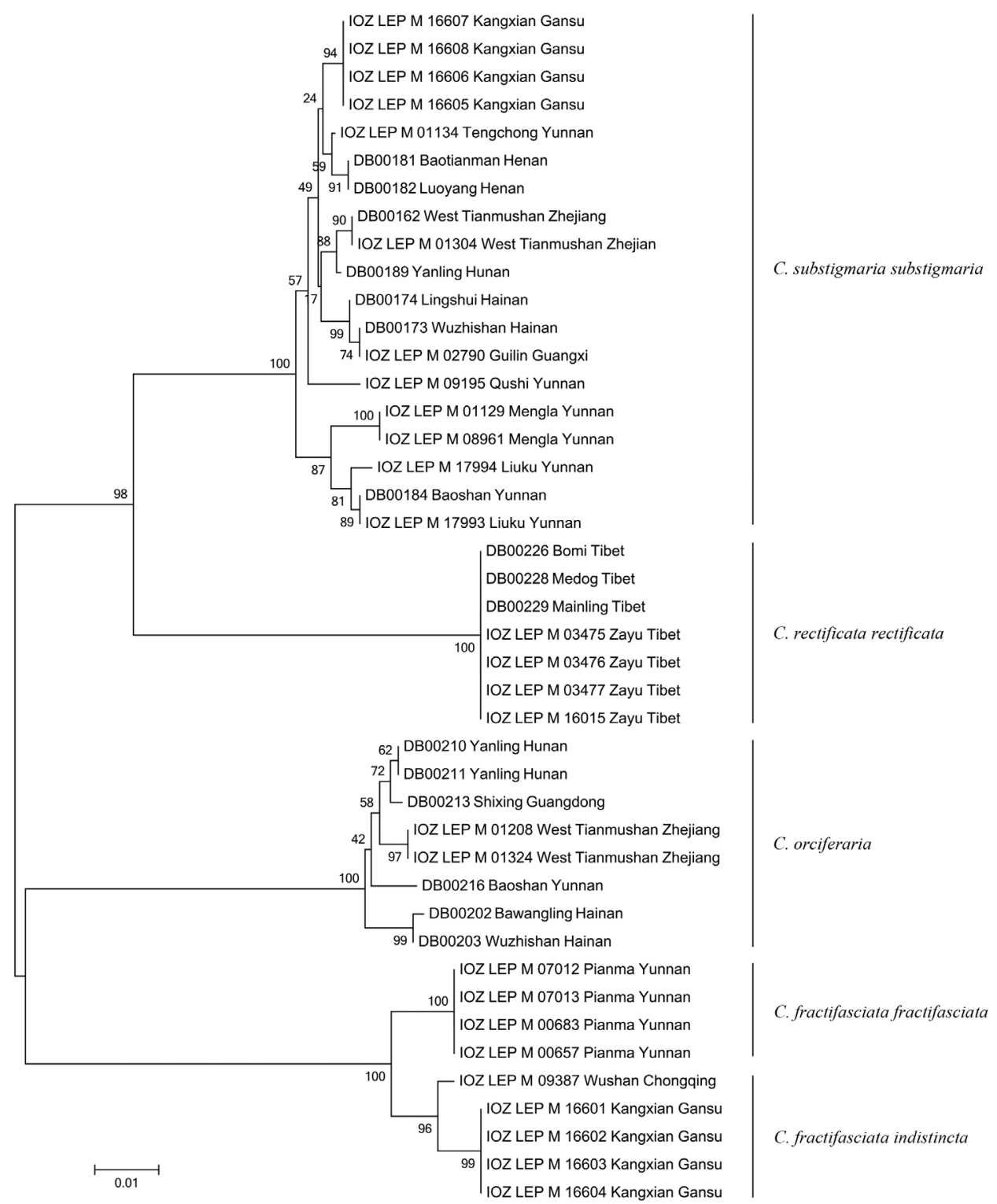

Figure 58. Neighbour joining tree (Kimura 2-parameter distance model for 658bp COI marker) for Chinese Cyclidia species. Terminals with sequence ID and collecting locality.

Additionally, some structures of the male genitalia (e.g. the shape of the valva) sometimes varied among individuals of $C$. substigmaria. Therefore, species have been delineated on the basis of a combination of data from morphology and DNA barcodes. In the molecular analysis, DNA barcodes proved to be very helpful. The interspecific divergence of Cyclidia species (minimum distance 8.8\%, maximum distance 13.9\%) was much larger than the $2 \%$ or $3 \%$ of the threshold for species diagnosis (Hebert et al. 
2003, Hebert et al. 2004a, Hebert et al. 2004b). The remarkably high interspecific divergence and low intraspecific divergence on average $1 \%$ (minimum distance $0.0 \%$, maximum distance $2.6 \%$ ) fully supports the morphological species concept.

\section{Acknowledgements}

We express our sincere thanks to Anthony Galsworthy, and the trustees and staff of the Natural History Museum, London for allowing examination of material under their care. We are grateful to all collectors whose contributions made our work possible. We appreciate the previous work of Dr. Song Wenhui in Chinese Cyclidia species, and the work of Ms. Yang Chao in preparing some specimens and photographs. This work was supported by the National Science Foundation of China (No. 30870320), the National Science Fund for Fostering Talents in Basic Research (NSFC-J1210002), the Ministry of Science and Technology of the People's Republic of China (MOST Grant No. 2011FY120200) and a grant from the Key Laboratory of the Zoological Systematics and Evolution of the Chinese Academy of Sciences (No. O529YX5105).

\section{References}

Aurivillius CHR (1894) Neue Spinner aus Asien. Entomologisk tidskrift 15: 169-177.

Beccaloni G, Scoble M, Kitching I, Simonsen T, Robinson G, Pitkin B, Hine A, Lyal C (Eds) (2003) The Global Lepidoptera Names Index (LepIndex). World Wide Web electronic publication. http://www.nhm.ac.uk/entomology/lepindex [accessed 26 Novermber 2015]

Bryk F (1943) Entomological results from the Swedish expedition 1934 to Burma and British India. Lepidoptera: Drepanidae. Arkiv för Zoologi Band 34A (13): 1-30.

Chang BS (1989) Illustrated moths of Taiwan 1. Taiwan Provincial Museum, Taipei, 242 pp. [In Chinese]

Chen YL (2011) Phylogenetic reconstruction of Cyclidiinae (Lepidoptera: Drepanidae). MS thesis, National Sun Yat-sen University, Kaohsiung, Taiwan. http://etd.lib.nsysu.edu.tw/ETDdb/ETD-search-c/getfile?URN=etd-0725111-051148\&filename=etd-0725111-051148. pdf [accessed 26 November 2015]

Chou I, Xiang H (1984) Studo de Drepanedoj el Yunnan Provinco (Lepidoptera: Drepanidae). Entomotaxonomia 6 (2-3): 159-169. [Abstract in Esperanto]

Chu HF, Wang LY (1987) Taxonomy and geographical distribution of Cyclidiidae. Acta Entomologica Sinica 30 (2): 203-211. [In Chinese]

Chu HF, Wang LY (1991) Fauna Sinica. Insecta. Vol. 3. Lepidoptera Cyclidiidae Drepanidae. Science Press, Beijing, vii+269 pp. [In Chinese]

Chu HF (Ed.) (1981) Iconocraphia Heterocerorum Sinicorum. Vol. I. Science Press, Beijing, iv+134 pp.+22 pp. (Index), 38 pls. [In Chinese]

Comstock JH (1918) The wings of insects. Comstock Publishing Company, Ithaca, New York, 430 pp. 
Cotes EC, Swinhoe C (1888) Geometrites. In: Cotes E, Swinhoe CC (Eds) A catalogue of the moths of India. Part. IV. Trustees of the Indian Museum, Calcutta, 463-590.

Fletcher DS (1979) Geometroidea. In: Nye WB (Ed.) The Generic Names of Moths of the World. Vol. 3. Trustees of the British Museum (Natural History), London, 243 pp.

Gaede M (1931) Family: Drepanidae. In: Strand E (Ed.) Lepidopterorum Catalogus. Vol. 49. W. Junk, Berlin, 60 pp.

Guenée A (1858) Uranides and Phalénites. In: Boisduval JBAD, Guenée A (Eds) Histoire naturelle des insectes: Spécies général des Lépidoptères. IX. Roret, Paris, 1-551. [In French]

Hajibabaei M, Janzen DH, Burns JM, Hallwachs W, Hebert PDN (2006) DNA barcodes distinguish species of tropical Lepidoptera. Proceedings of the National Academy of Sciences of the United States of America 103: 968-971. doi: 10.1073/pnas.0510466103

Hampson GF (1893) Illustrations of Typical Specimens of Lepidoptera Heterocera in the Collection of the British Museum. Part 9: The Macrolepidoptera Heterocera of Ceylon. Trustees of the British Museum (Natural History), London, v + 182 pp.

Hebert PDN, Cywinska A, Ball SL, deWaard JR (2003) Biological identifications through DNA barcodes. Philosophical Transactions of the Royal Society B: Biological Sciences 270: 313-321. doi: 10.1098/rspb.2002.2218

Hebert PDN, Penton EH, Burns JM (2004a) Ten species in one: DNA barcoding reveals cryptic species in the neotropical skipper butterfly Astraptes fulgerator. Proceedings of the National Academy of Sciences of the United States of America 101: 14812-14817.

Hebert PDN, Stoeckle MY, Zemlak TS, Francis CM (2004b) Identification of birds through DNA barcodes. Plos Biology 2: 1657-1663.

Holloway JD (1998) The Moths of Borneo. Part 8: Family Castniidae. Callidulidae. Drepanidae and Uraniidae. Malayan Nature Journal 52: 7-76.

Hübner J (1824-1831) Zuträge zur Sammlung exotischer Schmetterlinge, bestehend in Bekundigung einzelner Fliegmuster neuer oder rarer nichteuropäischer Gattungen 3. Im Verlag der Hübner'schen Werke bei C. Geyer, Augsburg, 103 pp.

Inoue H (1962) Lepidoptera: Cyclidiidae, Drepanidae. In: Inoue H (Ed.) Insecta Japonica. Vol. 2. Hokuryukan Publishing, Tokyo, 1-54, 1-3 pls. [In Japanese]

Inoue H (1992) Geometridae, Thyatiridae, Cyclidiidae, Drepanidae. In: Heppner JB, Inoue H. Lepidoptera of Taiwan. Volume. 1, part 2: Checklist. Association for Tropical Lepidoptera, Florida, 111-129, 151-153.

Jiang N, Liu SX, Xue DY, Tang MJ, Xiao Q, Han HX (2014) External morphology and molecular identification of two tea Geometrid moth from southern China. Chinese Journal of Applied Entomology 51: 987-1002. [In Chinese]

Kadoorie Farm, Botanic Garden (2004) Report of Rapid Biodiversity Assessments at Dachouding and Sanyue Nature Reserves, Northwest Guangdong, China, April 2001. South China Forest Biodiversity Survey Report Series (Online Simplified Version): No. 37. KFBG, Hong Kong SAR, ii+33 pp.

Kimura M (1980) A simple method for estimating evolutionary rates of base substitutions through comparative studies of nucleotide sequences. Journal of molecular evolution 16: 111-120. doi: 10.1007/BF01731581 
Kirby WF (1892) A Synonymic Catalogue of Lepidoptera Heterocera. R. Friedlander and Son., Berlin, $951 \mathrm{pp}$.

Klots AB (1970) Lepidoptera. In: Tuxen SL (Ed.) Taxonomist's Glossary of Genitalia in Insects. Munksgaard, Copenhagen, 115-130.

Kristensen NP (Ed.) (2003) Handbook of Zoology, Vol. IV. Arthropoda: Insecta. Part 36. Walter de Gruyter, Berlin, New York, 564 pp.

Leech JH (1897) On Lepidoptera Heterocera from China, Japan, and Corea. Annals and Magazine of Natural History (6) 19: 180-235. doi: 10.5962/bhl.title.22195

Leech JH (1898) Lepidoptera Heterocera from Northern China, Japan and Corea. Transactions of the Royal Entomological Society of London 46(3): 261-379.

Lutz W, Kobes R (2002) Cyclidia diehli sp. n. (Lepidoptera: Cyclidiinae) a species new to science. Heterocera Sumatrana 12: 177-183.

Meier R, Kwong S, Vaidya G, Ng PKL (2006) DNA barcoding and taxonomy in Diptera: a tale of high intraspecific variability and low identification success. Systematic Biology 55: 715-728. doi: 10.1080/10635150600969864

Meier R, Zhang GY, Ali1 F (2008) The use of mean instead of smallest interspecific distances exaggerates the size of the "Barcoding Gap" and leads to misidentification. Systematic Biology 57: 809-813. doi: 10.1080/10635150802406343

Minet J, Scoble MJ (1999) The drepanoid/geometroid Assemblage. In: Kristensen NP (Ed.) Lepidoptera, Moths and Butterflies. Vol. I: Evolution, Systematics, and Biogeography. Handbook of Zoology. Vol. IV, Arthropoda: Insecta, Part 35. Walter de Gruyter, Berlin and New York, 301-320.

Minet J (1983) Étude morphologique et phylogénétique des organs tympaniques des Pyraloidea. I. généralités et homologies. (Lep. Glossata). Annales de la Société entomologique de France (N.S.) 19: 175-207. [In French]

Minet J (2002) The Epicopeiidae: Phylogeny and a redefinition, with the description of new taxa (Lepidoptera: Drepanoidea). Annales de la Societe Entomologique de France 38: 463-487. doi: 10.1080/00379271.2002.10697355

Moore F (1886) List of the Lepidopterous Insects collected in Tavoy and in Siam during 1884 and 1885 by the Indian Museum Collector. Part i. Heterocera. Journal of the Asiatic Society of Bengal 55: 97-101.

Nichols SW (Ed.) (1989) The Torre-Bueno Glossary of Entomology. New York Entomological Society in cooperation with the American Museum of Natural History, New York, 840 pp.

Oberthür C (1912) Revision des Phalénites décrites par Guenée dans le species général des Lépidoptéres (Tome IX.). Famille II. Ennomidae, Guenée. Études de Lépidoptérologie Comparée 6: 223-307, 346-355. [In French]

Park KT, Kim M, Kwon YD, Ji EM (2011) A review of the genus Oreta Walker in Korea, with description of a new species (Lepidoptera: Drepanidae). Journal of Asia-Pacific Entomology 14(3): 311-316. doi: 10.1016/j.aspen.2011.04.001

Prout LB (1918) New moth species in the Joicey collection. Annals and Magazine of Natural History 9(11): 412-416.

Ratnasingham S, Hebert PDN (2007) BOLD: The Barcode of Life Data System (http://www.barcodinglife.org). Molecular Ecology Notes 7: 355-364. doi: 10.1111/j.1471-8286.2007.01678.x 
Saitou N, Nei M (1987) The neighbor-joining method, A new method for reconstructing phylogenetic trees. Molecular Biology and Evolution 4: 406-425.

Scoble MJ (1992) The Lepidoptera, Form, Function and Diversity. Oxford University Press, Oxford, xi+404 pp.

Sihvonen P, Skou P, Flamigni C, Fiumi G, Hausmann A (2014) Revision of the Hylaea fasciaria (Linnaeus, 1758) species group in the western Palaearctic (Lepidoptera: Geometridae, Ennominae). Zootaxa 3768(4): 469-486.

Song WH, Xue DY, Han HX (2011) A taxonomic revision of Tridrepana Swinhoe, 1895 in China, with descriptions of three new species (Lepidoptera, Drepanidae). Zootaxa 3021: 39-62.

Song WH, Xue DY, Han HX (2012) Revision of Chinese Oretinae (Lepidoptera, Drepanidae). Zootaxa 3445: 1-36.

Strand E (1911) Family: Drepanidae. In: Seitz A (Ed.) The Macrolepidoptera of the World. Vol. 2: the Palearctic Bombyces and Sphinges. Alfred Kernen, Stuttgart, 195-206.

Sugi S (1987) Larvae of Large Moths in Japan. Kodansha, Tokyo, 453 pp, 120 pls. [In Japanese] Swinhoe C (1899) New species of Oriental Lepidoptera. The Annals and Magazine of Natural History 7: 102-116. doi: 10.1080/00222939908678084

Tamura K, Peterson D, Peterson N, Stecher G, Nei M, Kumar S (2011) MEGA5: molecular evolutionary genetics analysis using likelihood, distance, and parsimony methods. Molecular Biology and Evolution 28: 2731-2739. doi: 10.1093/molbev/msr121

Walker F (1860) List of the specimens of Lepidopterous Insects in the collection of the British Museum. Part 20. Edward Newman, London, 1-276.

Walker F (1862a) List of the specimens of Lepidopterous Insects in the collection of the British Museum. Part 24. Edward Newman, London, 1021-1280.

Walker F (1862b) List of the specimens of Lepidopterous Insects in the collection of the British Museum. Part 25. Edward Newman, London, 1281-1477.

Walker F (1866) List of the specimens of Lepidopterous Insects in the collection of the British Museum. Part 35. Edward Newman, London, 1535-2040.

Warren W (1897) New genera and species of moths from the Old World regions in the Tring Museum. Novitates Zoologicae 4: 12-130.

Warren W (1901) Drepanulidae, Uraniidae, and Geometridae from the Palaearctic and IndoAustralian Regions. Novitates Zoologicae 8: 190-201.

Warren W (1901) Drepanulidae, Uraniidae, and Geometridae from the Palaearctic and IndoAustralian Regions. Novitates Zoologicae 8: 190-201.

Warren W (1914) New species of Drepanulidae, Noctuidae and Geometridae in the Tring Museum. Novitates Zoologicae 21: 401-425.

Warren W (1922-1928) Family: Drepanidae. In: Seitz A (Ed.) The Macrolepidoptera of the World. Vol. 10: Bombyces and Sphinges of the Indo-Australian Region. Alfred Kernen, Stuttgart, 443-490, pls. 48-50.

Wu CG, Han HX, Xue DY (2010) A pilot study on the molecular phylogeny of Drepanoidea (Insecta: Lepidoptera) inferred from the nuclear gene EF-1a and the mitochondrial gene COI. Bulletin of Entomological Research 100: 207-216. doi: 10.1017/S0007485309990162 
Yan SH, Chen YL, Wu SW (2009) Biota Taiwanica. Hexapoda: Lepidoptera, Drepanoidea, Drepanidae (Cyclidiinae). National Sun Yat-Sen University, Kaohsiung, 10 pp.

Yen SH, Robinson GS, Quicke DLJ (2005) The phylogenetic relationships of Chalcosiinae (Lepidoptera, Zygaenoidea, Zygaenidae). Zoological Journal of the Linnean Society 143: 161-341. doi: 10.1111/j.1096-3642.2005.00139.x

Yang Z, Landry JF, Handfield L, Zhang Y, Alma Solis M, Handfield D, Scholtens BG, Mutanen M, Nuss M, Hebert PDN (2012) DNA barcoding and morphology reveal three cryptic species of Anania (Lepidoptera: Crambidae: Pyraustinae) in North America, all distinct from their European counterpart. Systematic Entomology 37: 686-705. doi: 10.1111/j.1365-3113.2012.00637.x

Zhou TY, Wang LY (1985) A preliminary study on Cycidia substigmaria. Chinese Bulletin of Entomology 22 (3): 113-116. [In Chinese] 